\title{
An Energy-Preserving Scheme for the Coupled Gross-Pitaevskii Equations
}

\author{
Lan Wang ${ }^{1,2}$, Wenjun Cai ${ }^{1}$ and Yushun Wang ${ }^{1, *}$ \\ ${ }^{1}$ Jiangsu Collaborative Innovation Center of Biomedical Functional Materials, School of \\ Mathematical Sciences, Nanjing Normal University, Nanjing, Jiangsu 210023, China \\ 2 School of Mathematics and Statistics, Jiangxi Normal University, Nanchang, \\ Jiangxi 330022, China
}

Received 22 October 2019; Accepted (in revised version) 23 April 2020

\begin{abstract}
An energy-preserving scheme is proposed for the coupled Gross-Pitaevskii equations. The scheme is constructed by high order compact method in the spatial direction and average vector field method in the temporal direction, respectively. The scheme is energy-preserving, stable, and of sixth order in space and of second order in time. Numerical experiments verify the theoretical results. The dynamic behavior modeled by the coupled Gross-Pitaevskii equations is also numerically investigated.
\end{abstract}

AMS subject classifications: 65M06, 65M12, 65Z05, 70H15

Key words: Coupled Gross-Pitaevskii equations, average vector field method, high order compact method, energy-preserving scheme.

\section{Introduction}

In Bose-Einstein condensates (BECs) community, using mean field approximation, the two-component time-dependent dimensionless coupled Gross-Pitaevskii (CGP) equations with external potentials and effective Rabi frequencies is as follows [11, 16, 22, 23, $25,35]$

$$
\begin{aligned}
& i u_{t}=\left(-\frac{1}{2} \Delta+s_{1}(x, y)+\beta_{11}|u|^{2}+\beta_{12}|v|^{2}\right) u-L_{z} u-\lambda v, \quad(x, y) \in \mathbb{R}^{2}, \quad t>0, \\
& i v_{t}=\left(-\frac{1}{2} \Delta+s_{2}(x, y)+\beta_{21}|u|^{2}+\beta_{22}|v|^{2}\right) v-L_{z} v-\lambda u, \quad(x, y) \in \mathbb{R}^{2}, \quad t>0,
\end{aligned}
$$

where $i^{2}=-1$, and $\Delta$ is the Laplace operator. We only consider two-dimensional case, i.e., $\Delta=\partial_{x x}+\partial_{y y}$ in this paper. $u(x, y, t), v(x, y, t)$ are the complex-valued wave functions.

*Corresponding author.

Emails: w10908@yeah.net (L. Wang), caiwenjun@njnu.edu.cn (W. J. Cai), wangyushun@njnu.edu.cn (Y.S. Wang) 
$s_{l}(x, y)(l=1,2)$ are the real-valued external trapping potential functions. In the harmonic potentials, they are generally in the forms

$$
s_{l}(x, y)=\frac{1}{2}\left(\omega_{x, l}^{2} x^{2}+\omega_{y, l}^{2} y^{2}\right)
$$

with dimensionless frequencies $\omega_{x, l}$ and $\omega_{y, l}(l=1,2)$ in the $x$ and $y$ directions, respectively. The action between intra-component and inter-component atomic is represented by

$$
\beta_{l k}=\beta_{k l}=\frac{4 \pi N_{0} \alpha_{l k}}{\alpha_{0}}, \quad k, l=1,2,
$$

with $N_{0}$ being the total numbers of the particles in BECs, $\alpha_{0}$ being the dimensionless spatial unit, and $\alpha_{k l}=\alpha_{l k}$ being the s-wave scattering lengths between the $l$-th and $k$-th components (positive for repulsive interaction and negative for attractive interaction). $L_{z}$ is the rotating angular momentum with rotating speed $\Omega$, defined as

$$
L_{z}=i \Omega\left(y \partial_{x}-x \partial_{y}\right) \text {. }
$$

$\lambda$ is the effective Rabi frequency to realize the internal atomic Josephson junction by a Raman transition. If there is no effective Rabi frequency and no rotation, i.e., $\lambda=0$ and $\Omega=0$, the CGP equations collapse to the coupled nonlinear Schrödinger equations, they have been discussed by many authors [21].

Under appropriate assumption, to make the problem well-posed, with the following initial data

$$
u(x, y, 0)=u_{0}(x, y), \quad v(x, y, 0)=v_{0}(x, y),
$$

the problem (1.1a)-(1.2) satisfies the following laws [5].

- The component-masses

$$
\mathcal{M}_{u}(t)=\int_{\mathbb{R}^{2}}|u(x, y, t)|^{2} d x d y \quad \text { and } \quad \mathcal{M}_{v}(t)=\int_{\mathbb{R}^{2}}|v(x, y, t)|^{2} d x d y
$$

with respect to the $u$-component and $v$-component particles, respectively, satisfy the equalities

$$
\begin{aligned}
& \frac{d}{d t} \mathcal{M}_{u}(t)=-\lambda \int_{\mathbb{R}^{2}}(v(x, y, t) \overline{u(x, y, t)}-u(x, y, t) \overline{v(x, y, t)}) d x d y \\
& \frac{d}{d t} \mathcal{M}_{v}(t)=-\lambda \int_{\mathbb{R}^{2}}(u(x, y, t) \overline{v(x, y, t)}-v(x, y, t) \overline{u(x, y, t)}) d x d y .
\end{aligned}
$$

Therefore, the total mass of the particles of the system is an invariant, that is,

$$
\mathcal{M}(t)=\mathcal{M}_{u}(t)+\mathcal{M}_{v}(t)=\mathcal{M}(0) .
$$

Furthermore, $\mathcal{M}_{u}(t)=\mathcal{M}_{u}(0)$ and $\mathcal{M}_{v}(t)=\mathcal{M}_{v}(0)$ in case of $\lambda=0$. This indicates that the particle masses of each component are conserved. 
- The energy of the system is conserved, that is,

$$
\begin{aligned}
\mathcal{E}(t) & =\int_{\mathbb{R}^{2}}\left[\frac{1}{2}|\nabla w|^{2}+s^{T}|w|^{2}-w^{T} L_{z} \bar{w}+\frac{1}{2}\left(|w|^{2}\right)^{T} \beta|w|^{2}-\lambda w^{T} \bar{w}^{\perp}\right] d x d y \\
& =\mathcal{E}(0)
\end{aligned}
$$

where

$$
\begin{aligned}
& w(x, y, t)=\left[\begin{array}{l}
u(x, y, t) \\
v(x, y, t)
\end{array}\right], \quad s(x, y)=\left[\begin{array}{l}
s_{1}(x, y) \\
s_{2}(x, y)
\end{array}\right], \quad \beta=\left[\begin{array}{ll}
\beta_{11} & \beta_{12} \\
\beta_{21} & \beta_{22}
\end{array}\right], \\
& |w|^{2}=\left[\begin{array}{l}
|u|^{2} \\
|v|^{2}
\end{array}\right], \quad w \bar{w}^{\perp}=u \bar{v}+v \bar{u} .
\end{aligned}
$$

A lot of efficient numerical methods have been proposed to study the GP equation with/without the angular momentum rotation terms, including finite difference methods $[4,9,12,15,18,33,34]$, time-splitting spectral methods $[3,5,6,26,31]$. Of course, all of these methods have their advantages and disadvantages. For example, finite difference methods are easily to deal with variable coefficients problems, but there are additional troubles for irregular domains. Spectral methods have exponential convergence rate for smooth problems, but are difficult to deal with the angular momentum terms because they are spatial variables dependent. Splitting methods are easily to deal with the multidimensional problems [27], but often destroy the essential structure of the original system, such as energy, mass. This motivates us to construct high accuracy and structurepreserving schemes for the CGP equations.

The tendency towards highly accurate numerical methods for partial differential equations (PDEs) has excited a renewed interest recently, especially accurate methods with small stencils, i.e., high order compact (HOC) method. This kind of numerical method features high accuracy (at least second order higher than general finite difference schemes), small stencil (less nodes needed to obtain the same order scheme comparing to general finite difference schemes) [20,21]. Moreover, as pointed out by Adam, their truncation errors are about four to six times smaller than the same order noncompact schemes [1]. They are favored in various differential equations, such as parabolic type equations [19], Schrödinger-type equations [12,14,21,30,32].

As is well known, the energy conservation law is an important and intrinsic property for an autonomous Hamiltonian system [29]. Therefore, it is necessary and significant to develop energy-preserving numerical schemes for the CGP equations. However, most of the existing literatures ignore the energy structure of the CGP equations, especially in multidimensional case. In this paper, we intend to propose an energy-preserving scheme for the CGP equations by the average vector field (AVF) method which was introduced by Quispel et al. recently [24,28]. One of the excellent advantages of the AVF method is that it can preserve the energy invariant automatically $[7,8,13]$. Moreover, it is relatively easy to construct temporal high order energy-preserving schemes for Hamiltonian systems. 
We discretize the CGP equations with HOC method in the spatial direction and AVF method in the temporal direction, respectively.

This paper is organized as follows. In Section 2, we present an HOC-AVF scheme for the CGP equations obtained by HOC method in space and AVF method in time. By theoretical analysis, we find the scheme is energy-preserving, of sixth order in space and second order in time. The convergence of this scheme is investigated in Section 3. In Section 4, some numerical experiments are reported to verify the theoretical analysis. Finally, some conclusions and remarks are given in Section 5.

\section{An HOC-AVF scheme for the CGP equations}

In this section, we propose an HOC-AVF scheme for the CGP equations (1.1a)-(1.1b). In practical numerical computing, we always truncate the whole plane $\mathbb{R}^{2}$ into a large enough but bounded rectangle $D=\left[x_{l}, x_{r}\right] \times\left[y_{l}, y_{r}\right]$. For simplicity, we numerically study the CGP equations (1.1a)-(1.1b) constrained by periodic boundary condition. The scheme and its theoretical results can be extended to general homogeneous Dirichlet boundary condition with a minor modification.

Before developing the numerical scheme, we give some notations: $x_{j}=x_{l}+j h_{x}$, $y_{k}=y_{l}+k h_{y}, t_{n}=n \tau(j=0,1, \cdots J ; k=0,1, \cdots, K ; n=0,1, \cdots, N)$ with $h_{x}=\left(x_{r}-x_{l}\right) / J$, $h_{y}=\left(y_{r}-y_{l}\right) / K, \tau=T / N$. We define the index sets $S_{p}=\{(j, k) \mid j=1, \cdots, J ; k=1, \cdots, K\}$, $\mathrm{T}_{\mathrm{N}}=\{n \mid n=0,1, \cdots, N\}$, and the periodic function space

$$
X_{\mathrm{p}}=\left\{u=\left(u_{j k}\right)_{(j, k) \in \mathrm{S}_{\mathrm{p}}} \mid u_{j k}=u_{J+j, k}, u_{j k}=u_{j, K+k}\right\} \subseteq \mathbb{C}^{J \times K} .
$$

We denote the exact solutions

$$
U_{j k}^{n}=u\left(x_{j}, y_{k}, t_{n}\right), \quad V_{j k}^{n}=v\left(x_{j}, y_{k}, t_{n}\right),
$$

and approximate solutions

$$
\begin{aligned}
& u_{j k}^{n} \approx u\left(x_{j}, y_{k}, t_{n}\right), \quad v_{j k}^{n} \approx v\left(x_{j}, y_{k}, t_{n}\right), \quad \delta_{t} u_{j k}^{n}=\frac{u_{j k}^{n+1}-u_{j k}^{n}}{\tau}, \\
& \delta_{x}^{+} u_{j k}=\frac{u_{j+1, k}-u_{j k}}{h_{x}}, \quad \delta_{y}^{+} u_{j k}=\frac{u_{j, k+1}-u_{j k}}{h_{y}}, \\
& \delta_{x}^{2} u_{j k}=\frac{u_{j+1, k}-2 u_{j k}+u_{j-1, k}}{h_{x}^{2}}, \quad \delta_{y}^{2} u_{j k}=\frac{u_{j, k+1}-2 u_{j k}+u_{j, k-1}}{h_{y}^{2}}, \quad h=\max \left\{h_{x}, h_{y}\right\} .
\end{aligned}
$$

We define inner product and associated norms, for vectors $u, v, \phi, \varphi \in \mathrm{X}_{p}$,

$$
\langle u, v\rangle=h_{x} h_{y} \sum_{j=1}^{J} \sum_{k=1}^{K} u_{j k} \bar{v}_{j k}, \quad\|u\|=\sqrt{\langle u, u\rangle}, \quad\langle(u, v),(\varphi, \phi)\rangle=\langle u, \varphi\rangle+\langle v, \phi\rangle,
$$




$$
\begin{aligned}
& \|(u, v)\|=\sqrt{\langle u, u\rangle+\langle v, v\rangle}, \quad\|u\|_{p}=\left(h_{x} h_{y} \sum_{j=1}^{J} \sum_{k=1}^{K}\left|u_{j k}\right|^{p}\right)^{1 / p}, \quad\|u\|_{\infty}=\max _{(j, k) \in \mathrm{S}_{p}}\left|u_{j k}\right|, \\
& |u|_{1}=\left\|\nabla_{h} u\right\|=\sqrt{\left\|\delta_{x}^{+} u\right\|^{2}+\left\|\delta_{y}^{+} u\right\|^{2}}, \quad|(u, v)|_{1}=\sqrt{|u|_{1}^{2}+|v|_{1}^{2}} .
\end{aligned}
$$

To construct the HOC-AVF scheme, we first review the HOC method and the AVF method.

\subsection{High order compact method}

High order compact (HOC) method is a kind of efficient numerical method to approximate derivatives. It discretizes the derivatives with the fewest nodes to get the expected accuracy. In order to get higher accuracy with fewer nodes, we solve the derivatives implicitly rather than explicitly. The basic idea lying in this kind of method is to equate a linear combination of derivative values at adjacent nodes to a linear combination of function values at the adjacent nodes. Then we can determine the combination coefficients according to the power of step size $h$. To make the accuracy as high as possible, here we use symmetric stencil and symmetric coefficients. Furthermore, this kind of choice can reduce the dissipation of the scheme. We will detail this idea for $u^{\prime}\left(x_{j}\right)$ and $u^{\prime \prime}\left(x_{j}\right)$ with sixth order HOC method.

For $u^{\prime}\left(x_{j}\right)$, we start from an implicit discretization in the form $[1,14,20]$

$$
\alpha_{1} u_{j-1}^{\prime}+u_{j}^{\prime}+\alpha_{1} u_{j+1}^{\prime}=b_{1} \frac{u_{j+2}-u_{j-2}}{4 h}+a_{1} \frac{u_{j+1}-u_{j-1}}{2 h}, \quad j=1, \cdots, J,
$$

where $a_{1}, b_{1}$ and $\alpha_{1}$ are undetermined parameters which depend on the accuracy constraints. By Taylor expansions of all the involved arguments $u_{j}, u_{j}^{\prime}$, pairing the series according to the power of $h$, we obtain

$$
\begin{cases}a_{1}+b_{1}=1+2 \alpha_{1}, & \text { second-order } \\ a_{1}+2^{2} b_{1}=2 \frac{3 !}{2 !} \alpha_{1}, & \text { fourth-order } \\ a_{1}+2^{4} b_{1}=2 \frac{5 !}{4 !} \alpha_{1}, & \text { sixth-order. }\end{cases}
$$

Now it yields a sixth order approximation to $u^{\prime}\left(x_{j}\right)$ with

$$
\alpha_{1}=1 / 3, \quad a_{1}=14 / 9, \quad b_{1}=1 / 9,
$$

whose dominant part of the truncation error is $\frac{4}{7 !} \frac{d^{7} u\left(x_{j}\right)}{d x^{7}} h^{6}$. Under the periodic boundary condition, the approximation to $u^{\prime}\left(x_{j}\right)$ can be written in the matric form

$$
A_{x} \mathbf{u}^{\prime}=B_{x} \mathbf{u} \Rightarrow \mathbf{u}^{\prime}=D_{x} \mathbf{u},
$$


where $D_{x}=A_{x}^{-1} B_{x}$, and

$$
A_{x}=\frac{1}{5}\left[\begin{array}{ccccc}
3 & 1 & & & 1 \\
1 & 3 & 1 & & \\
& \ddots & \ddots & \ddots & \\
& & 1 & 3 & 1 \\
1 & & & 1 & 3
\end{array}\right], \quad B_{x}=\frac{1}{60 h}\left[\begin{array}{cccccc}
0 & 28 & 1 & & -1 & -28 \\
-28 & 0 & 28 & 1 & & -1 \\
-1 & -28 & 0 & 28 & 1 & \\
& \ddots & \ddots & \ddots & \ddots & \ddots \\
& -1 & -28 & 0 & 28 & 1 \\
1 & & -1 & -28 & 0 & 28 \\
28 & 1 & & -1 & -28 & 0
\end{array}\right] .
$$

In a similar way, we can derive an approximation to $u^{\prime \prime}\left(x_{j}\right)$ with sixth order as follows

$$
\alpha_{2} u_{j-1}^{\prime \prime}+u_{j}^{\prime \prime}+\alpha_{2} u_{j+1}^{\prime \prime}=b_{2} \delta_{2 x}^{2} u_{j}+a_{2} \delta_{x}^{2} u_{j}
$$

where $\delta_{2 x}^{2} u_{j}=\frac{u_{j+2}-2 u_{j}+u_{j-2}}{4 h^{2}}$. The coefficients satisfy the following equations

$$
\begin{cases}a_{2}+b_{2}=1+2 \alpha_{2}, & \text { second-order } \\ a_{2}+2^{2} b_{2}=\frac{4 !}{2 !} \alpha_{2}, & \text { fourth-order } \\ a_{2}+2^{4} b_{2}=\frac{6 !}{4 !} \alpha_{2}, & \text { sixth-order. }\end{cases}
$$

The unique solution of Eq. (2.5) is

$$
\alpha_{2}=2 / 11, \quad a_{2}=12 / 11, \quad b_{2}=3 / 11 .
$$

Under the periodic boundary conditions, the approximation to $u^{\prime \prime}\left(x_{j}\right)$ can be written in the matric form

$$
A_{x x} \mathbf{u}^{\prime \prime}=B_{x x} \delta_{x}^{2} \mathbf{u} \Rightarrow \mathbf{u}^{\prime \prime}=D_{x x} \delta_{x}^{2} \mathbf{u},
$$

where $D_{x x}=A_{x x}^{-1} B_{x x}$. With some algebraic operation, we can know that $A_{x x}$ and $B_{x x}$ can be written in the form

$$
A_{x x}=\frac{1}{15}\left[\begin{array}{ccccc}
11 & 2 & & & 2 \\
2 & 11 & 2 & & \\
& \ddots & \ddots & \ddots & \\
& & 2 & 11 & 2 \\
2 & & & 2 & 11
\end{array}\right], \quad B_{x x}=\frac{1}{20}\left[\begin{array}{ccccc}
18 & 1 & & & 1 \\
1 & 18 & 1 & & \\
& \ddots & \ddots & \ddots & \\
& & 1 & 18 & 1 \\
1 & & & 1 & 18
\end{array}\right] \text {. }
$$

Remark 2.1. It is obvious that the matrices $A_{x}, A_{x x}, B_{x x}, D_{x x}$ are circulant and symmetric, while $B_{x}, D_{x}$ are circulant and skew-symmetric. They are all real. This kind of matrices have a lot of properties which are very useful during theoretical derivation. For example, circulant matrices are communicative, i.e., $A_{x} B_{x}=B_{x} A_{x}$. Moreover, $A_{x x}$ and $D_{x x}$ are 
positive defined matrices. It is able to be decomposed into $D_{x x}=C_{x}{ }^{T} C_{x}$. The similar results for those matrices for $y$-direction, $D_{y y}=C_{y}{ }^{T} C_{y}$. Therefore, we can define a new semi-norm of $u$ and $(u, v)$ by $D_{x x}$ and $D_{y y}$

$$
\begin{aligned}
& \widetilde{|u|_{1}}=\widetilde{\left\|\nabla_{h} u\right\|}=\sqrt{\left\langle-D_{x x} \delta_{x}^{2} u, u\right\rangle+\left\langle-D_{y y} \delta_{y}^{2} u, u\right\rangle}=\sqrt{\left\|C_{x} \delta_{x}^{+} u\right\|^{2}+\left\|C_{y} \delta_{y}^{+} u\right\|^{2}}, \\
& \widetilde{|(u, v)|_{1}}=\sqrt{|\widetilde{u}|_{1}^{2}+\widetilde{|v|_{1}^{2}}} .
\end{aligned}
$$

As we will prove that this semi-norm is equivalent to $|u|_{1}=\left\|\nabla_{h} u\right\|$.

\subsection{Average vector field method}

We consider a classical Hamiltonian system

$$
\frac{d z}{d t}=f(z)=S \nabla H(z), \quad z(0)=z_{0}, \quad z \in \mathbb{R}^{2 m},
$$

where $S$ is a skew-symmetric constant matrix, and $H: \mathbb{R}^{2 m} \rightarrow \mathbb{R}$ is the Hamiltonian energy functional. The Hamiltonian system (2.7) satisfies

$$
\frac{d H(z(t))}{d t}=\nabla H(z)^{T} f(z)=\nabla H(z)^{T} S \nabla H(z)=0 .
$$

This implies that along the solution trajectory the Hamiltonian energy functional $H(z)$ for the system (2.7) is independent of time $t$. As is well known, a numerical scheme that can preserve the discrete version of this property is attractive. AVF method is starting from this consideration. An AVF method for the Hamiltonian system (2.7) is defined by [28]

$$
\frac{z^{n+1}-z^{n}}{\tau}=\int_{0}^{1} f\left(\xi z^{n+1}+(1-\xi) z^{n}\right) d \xi=S \int_{0}^{1} \nabla H\left(\xi z^{n+1}+(1-\xi) z^{n}\right) d \xi,
$$

where $\tau$ denotes the time step.

Theorem 2.1. The AVF method (2.8) preserves the energy exactly, i.e.,

$$
H^{n+1}=H\left(z^{n+1}\right)=H^{n}=\cdots=H^{0} .
$$

Proof. By straightforward computing and using the AVF method (2.8), we have

$$
\begin{aligned}
& \frac{H\left(z^{n+1}\right)-H\left(z^{n}\right)}{\tau} \\
= & \frac{1}{\tau} \int_{0}^{1} \frac{d}{d \xi} H\left(\xi z^{n+1}+(1-\xi) z^{n}\right) d \xi \\
= & \left(\int_{0}^{1} \nabla H\left(\xi z^{n+1}+(1-\xi) z^{n}\right) d \xi\right)^{T} \frac{z^{n+1}-z^{n}}{\tau} \\
= & \left(\int_{0}^{1} \nabla H\left(\xi z^{n+1}+(1-\xi) z^{n}\right) d \xi\right)^{T} S \int_{0}^{1} \nabla H\left(\xi z^{n+1}+(1-\xi) z^{n}\right) d \xi \\
= & 0 .
\end{aligned}
$$


This suggests that the discrete energy is invariant, i.e.,

$$
H^{n+1}=H^{n} \text {. }
$$

The proof is completed.

\subsection{Numerical scheme for the CGP equations}

Now, we discretize the CGP equations (1.1a)-(1.1b) in the spatial direction by the sixth order HOC schemes (2.3) and (2.6), which result in

$$
\begin{aligned}
& i \frac{d}{d t} u_{j k}=\left[-\frac{1}{2}\left(\mathcal{D}_{x x} \delta_{x}^{2}+\mathcal{D}_{y y} \delta_{y}^{2}\right)+s_{1 j k}-L_{h, z j, k}+\left(\beta_{11}\left|u_{j k}\right|^{2}+\beta_{12}\left|v_{j k}\right|^{2}\right)\right] u_{j k}-\lambda v_{j k}, \\
& i \frac{d}{d t} v_{j k}=\left[-\frac{1}{2}\left(\mathcal{D}_{x x} \delta_{x}^{2}+\mathcal{D}_{y y} \delta_{y}^{2}\right)+s_{2 j k}-L_{h, z j, k}+\left(\beta_{21}\left|u_{j k}\right|^{2}+\beta_{22}\left|v_{j k}\right|^{2}\right)\right] v_{j k}-\lambda u_{j k},
\end{aligned}
$$

where $L_{h, z j, k}=i \Omega\left(y_{k} \mathcal{D}_{x}-x_{j} \mathcal{D}_{y}\right)$, and $\mathcal{D}_{x}, \mathcal{D}_{y}, \mathcal{D}_{x x}, \mathcal{D}_{y y}$ are the corresponding operators of the matrices $D_{x}, D_{y}, D_{x x}, D_{y y}$.

Then we discretize Eqs. (2.10a)-(2.10b) in time by the AVF method (2.8) and have

$$
\begin{aligned}
i \delta_{t} u_{j k}^{n}=- & \frac{1}{2}\left(\mathcal{D}_{x x} \delta_{x}^{2}+\mathcal{D}_{y y} \delta_{y}^{2}\right) u_{j k}^{n+\frac{1}{2}}+s_{1 j k} u_{j k}^{n+\frac{1}{2}}-L_{h, z j, k} u_{j k}^{n+\frac{1}{2}}-\lambda v_{j k}^{n+\frac{1}{2}} \\
& +\frac{\beta_{11}}{6}\left(\left|u_{j k}^{n}\right|^{2} u_{j k}^{n}+\left|u_{j k}^{n+1}\right|^{2} u_{j k}^{n+1}+4\left|u_{j k}^{n+\frac{1}{2}}\right|^{2} u_{j k}^{n+\frac{1}{2}}\right) \\
& +\frac{\beta_{12}}{6}\left(\left|v_{j k}^{n}\right|^{2} u_{j k}^{n}+\left|v_{j k}^{n+1}\right|^{2} u_{j k}^{n+1}+4\left|v_{j k}^{n+\frac{1}{2}}\right|^{2} u_{j k}^{n+\frac{1}{2}}\right), \\
i \delta_{t} v_{j k}^{n}=- & \frac{1}{2}\left(\mathcal{D}_{x x} \delta_{x}^{2}+\mathcal{D}_{y y} \delta_{y}^{2}\right) v_{j k}^{n+\frac{1}{2}}+s_{2 j k} v_{j k}^{n+\frac{1}{2}}-L_{h, z j, k} v_{j k}^{n+\frac{1}{2}}-\lambda u_{j k}^{n+\frac{1}{2}} \\
& +\frac{\beta_{21}}{6}\left(\left|u_{j k}^{n}\right|^{2} v_{j k}^{n}+\left|u_{j k}^{n+1}\right|^{2} v_{j k}^{n+1}+4\left|u_{j k}^{n+\frac{1}{2}}\right|^{2} v_{j k}^{n+\frac{1}{2}}\right) \\
& +\frac{\beta_{22}}{6}\left(\left|v_{j k}^{n}\right|^{2} v_{j k}^{n}+\left|v_{j k}^{n+1}\right|^{2} v_{j k}^{n+1}+4\left|v_{j k}^{n+\frac{1}{2}}\right|^{2} v_{j k}^{n+\frac{1}{2}}\right),
\end{aligned}
$$

where $u_{j k}^{n+\frac{1}{2}}=\frac{1}{2}\left(u_{j k}^{n}+u_{j k}^{n+1}\right)$, etc. The initial data are discretized by

$$
u_{j k}^{0}=u_{0}\left(x_{j}, y_{k}\right), \quad v_{j k}^{0}=v_{0}\left(x_{j}, y_{k}\right) .
$$

The scheme (2.11a)-(2.11b) can be reformed as a vector form

$$
\begin{aligned}
i \delta_{t} w^{n}=- & \frac{1}{2}\left(\mathcal{D}_{x x} \delta_{x}^{2}+\mathcal{D}_{y y} \delta_{y}^{2}\right) w^{n+\frac{1}{2}}+s \cdot w^{n+\frac{1}{2}}-L_{h, z j, k} w^{n+\frac{1}{2}}-\lambda J w^{n+\frac{1}{2}} \\
& +\frac{1}{6}\left\{\left(\left|w^{n}\right|_{\beta}^{2} \cdot w^{n}+\left|w^{n+1}\right|_{\beta}^{2} \cdot w^{n+1}\right)+4\left|w^{n+\frac{1}{2}}\right|_{\beta}^{2} \cdot w^{n+\frac{1}{2}}\right\},
\end{aligned}
$$


where

$$
\begin{aligned}
& J=\left[\begin{array}{ll}
0 & 1 \\
1 & 0
\end{array}\right], \quad\left|w^{n}\right|^{2}=\left[\begin{array}{c}
\left|u^{n}\right|^{2} \\
\left|v^{n}\right|^{2}
\end{array}\right], \\
& \left|w^{n}\right|_{\beta}^{2}=\beta\left[\begin{array}{l}
\left|u^{n}\right|^{2} \\
\left|v^{n}\right|^{2}
\end{array}\right]=\left[\begin{array}{l}
\beta_{11}\left|u^{n}\right|^{2}+\beta_{12}\left|v^{n}\right|^{2} \\
\beta_{21}\left|u^{n}\right|^{2}+\beta_{22}\left|v^{n}\right|^{2}
\end{array}\right] .
\end{aligned}
$$

The "." product denotes the Hadamard product, which takes point-wise product of two vectors with the same size, i.e., $P \cdot Q=\left(p_{1} q_{1}, p_{2} q_{2}, \cdots, p_{n} q_{n}\right)^{T}$ if $P, Q \in \mathbb{C}^{n}$.

By Taylor's expansion, we know the HOC-AVF scheme (2.11a)-(2.11b) is of sixth order in space and of second order in time.

\section{Numerical analysis}

In this section, we investigate the HOC-AVF scheme (2.11a)-(2.11b) theoretically, mainly concerning on its global convergent rate.

First of all, according to the designing procedure, we have the following discrete energy conservation law.

Theorem 3.1. The HOC-AVF scheme (2.11a)-(2.11b) of the CGP equations (1.1a)-(1.1b) preserves the energy exactly, i.e.,

$$
\mathcal{H}^{n}=\mathcal{H}^{n-1}=\cdots=\mathcal{H}^{0}, \quad n=1, \cdots, N,
$$

where

$$
\begin{aligned}
\mathcal{H}^{n}= & \frac{1}{2}\left\|\left(\widetilde{\nabla_{h}} \widetilde{u^{n}, \nabla_{h}} v^{n}\right)\right\|^{2}+\left(\left\|u^{n}\right\|_{s_{1}}^{2}+\left\|v^{n}\right\|_{s_{2}}^{2}\right)+\frac{\beta_{11}}{2}\left\|u^{n}\right\|_{4}^{4} \\
& +\beta_{12}\left\langle\left|u^{n}\right|^{2},\left|v^{n}\right|^{2}\right\rangle+\frac{\beta_{22}}{2}\left\|v^{n}\right\|_{4}^{4}-2 \lambda \mathcal{R}\left(\left\langle u^{n}, v^{n}\right\rangle\right)-\left(\left\langle L_{h, z} u^{n}, u^{n}\right\rangle+\left\langle L_{h, z} v^{n}, v^{n}\right\rangle\right)
\end{aligned}
$$

with the weighted 2-norm

$$
\left\|u^{n}\right\|_{s_{1}}^{2}=h_{x} h_{y} \sum_{j, k} s_{1 j k}\left|u_{j k}^{n}\right|^{2} \quad \text { and } \quad\left\|v^{n}\right\|_{s_{2}}^{2}=h_{x} h_{y} \sum_{j, k} s_{2 j k}\left|v_{j k}^{n}\right|^{2} .
$$

Here $\mathcal{R}(\cdot)$ means taking real part from a given complex number.

Theorem 3.2. The HOC-AVF scheme (2.11a)-(2.11b) of the CGP equations (1.1a)-(1.1b) admits the following implicit mass conservation law:

$$
\begin{gathered}
\frac{\mathcal{M}^{n+1}-\mathcal{M}^{n}}{\tau}=\frac{h_{x} h_{y}}{12} \sum_{j, k}\left\{\left[\beta_{11} \frac{\left|u_{j k}^{n+1}\right|^{2}-\left|u_{j k}^{n}\right|^{2}}{\tau}+\beta_{12} \frac{\left|v_{j k}^{n+1}\right|^{2}-\left|v_{j k}^{n}\right|^{2}}{\tau}\right] \frac{u_{j k}^{n+1} \overline{u_{j k}^{n}}-u_{j k}^{n} \overline{u_{j k}^{n+1}}}{\tau}\right. \\
\left.+\left[\beta_{12} \frac{\left|u_{j k}^{n+1}\right|^{2}-\left|u_{j k}^{n}\right|^{2}}{\tau}+\beta_{22} \frac{\left|v_{j k}^{n+1}\right|^{2}-\left|v_{j k}^{n}\right|^{2}}{\tau}\right] \frac{v_{j k}^{n+1} \overline{v_{j k}^{n}}-v_{j k}^{n} \overline{v_{j k}^{n+1}}}{\tau}\right\} \tau^{2},
\end{gathered}
$$

where $\mathcal{M}^{n}=\left\|u^{n}\right\|^{2}+\left\|v^{n}\right\|^{2}$. 
Proof. This implicit mass conservation law can be verified direction by taking inner product of scheme (2.11a)-(2.11b) with $u^{n+\frac{1}{2}}, v^{n+\frac{1}{2}}$ and taking the imaginary part.

Proposition 3.1. If the system (1.1a)-(1.1b) is without rotation and without Josephson junction, i.e., $\Omega=\lambda=0$, and the interaction constant matrix $\beta$ is a non-negative or positive semi-definite matrix, then the solutions of the scheme (2.11a)-(2.11b) are bounded in the $l^{2}$ norm. In other words, there exists a positive genetic constant $C$ such that

$$
\left\|u^{n}\right\| \leq C, \quad\left\|v^{n}\right\| \leq C, \quad n \in T_{N} .
$$

Proof. By the discrete energy conservation law (3.1), if $\Omega=\lambda=0$, we have

$$
\max \left\{\left\|u^{n}\right\|^{2},\left\|v^{n}\right\|^{2}\right\} \leq C \mathcal{H}^{n}=C \mathcal{H}^{0}=C .
$$

Here we have used the fact that every term in $\mathcal{H}^{n}$ is non-negative if $\Omega=\lambda=0$.

Theorem 3.3. For any given $w^{n}=\left[u^{n}, v^{n}\right]^{T} \in X_{p} \times X_{p}$, there exists a solution

$$
w^{n+1}=\left[u^{n+1}, v^{n+1}\right]^{T} \in X_{p} \times X_{p}
$$

of the HOC-AVF scheme (2.11a)-(2.11b).

Proof. First, for any given $w^{n} \in X_{p} \times X_{p}$, we rewrite vector form (2.13) as

$$
w^{n+\frac{1}{2}}=w^{n}-\frac{\tau}{2} i\left(\mathcal{L} w^{n+\frac{1}{2}}+\frac{1}{6} \mathcal{N}\left(w^{n+\frac{1}{2}}\right)\right)
$$

where

$$
\begin{aligned}
& \mathcal{L}=-\frac{1}{2}\left(\mathcal{D}_{x x} \delta_{x}^{2}+\mathcal{D}_{y y} \delta_{y}^{2}\right)+s \cdot-L_{h, z j, k}-\lambda J \\
& \mathcal{N}\left(w^{n+\frac{1}{2}}\right)=\left(\left|w^{n}\right|_{\beta}^{2} \cdot w^{n}+\left|2 w^{n+\frac{1}{2}}-w^{n}\right|_{\beta}^{2} \cdot\left(2 w^{n+\frac{1}{2}}-w^{n}\right)\right)+4\left|w^{n+\frac{1}{2}}\right|_{\beta}^{2} \cdot w^{n+\frac{1}{2}} .
\end{aligned}
$$

Define a mapping $\Phi: X_{p} \times X_{p} \rightarrow X_{p} \times X_{p}$ as

$$
\Phi(w)=w-w^{n}+\frac{\tau}{2} i\left(\mathcal{L} w+\frac{1}{6} \mathcal{N}(w)\right) .
$$

It is obvious that $\Phi$ is continuous from $X_{p} \times X_{p}$ to $X_{p} \times X_{p}$. Taking inner product of Eq. (3.6) with $w$, one has

$$
\langle\Phi(w), w\rangle=\langle w, w\rangle-\left\langle w^{n}, w\right\rangle+\frac{\tau}{2} i\left\langle\left(\mathcal{L} w+\frac{1}{6} \mathcal{N}(w)\right), w\right\rangle .
$$

It is apparently that the third part in the right side of the above equality is a purely imaginary number. Therefore, the real part of Eq. (3.7) is

$$
\mathcal{R}(\langle\Phi(w), w\rangle)=\|w\|^{2}-\mathcal{R}\left(\left\langle w^{n}, w\right\rangle\right) \geq\|w\|^{2}-\|w\|\left\|w^{n}\right\| .
$$


This indicates that

$$
\lim _{\|w\| \rightarrow \infty} \frac{|\langle\Phi(w), w\rangle|}{\|w\|}=\infty
$$

Thus, $\Phi$ is a surjective mapping. It is easy to derive that there exists a solution $w^{n+\frac{1}{2}}$ satisfying $\Phi\left(w^{n+\frac{1}{2}}\right)=0$ by Brouwer fixed point theorem [17].

Lemma 3.1 (Gronwall's Inequality, [36]). Suppose that the discrete function $\left\{w^{n} \mid n=1, \cdots\right.$, $N ; N \tau=T\}$ satisfies the inequality

$$
w^{n}-w^{n-1} \leq A \tau w^{n}+B \tau w^{n-1}+C_{n} \tau
$$

where $A, B$ and $C_{n}(n=1, \cdots, N)$ are nonnegative constants. Then

$$
\max _{1 \leq n \leq N}\left|w^{n}\right| \leq\left(w^{0}+\tau \sum_{l=1}^{N} C_{l}\right) e^{2(A+B) T},
$$

where $\tau$ is sufficiently small, such that

$$
(A+B) \tau \leq \frac{N-1}{2 N}, \quad(N>1) .
$$

Lemma 3.2. For any complex functions $U, V, W, u, v, w \in X_{p}$, one has

$$
\begin{aligned}
& U V W-u v w=V W e_{u}+u W e_{v}+u v e_{w} \\
= & U W e_{v}+U V e_{w}+V W e_{u}-U e_{v} e_{w}-V e_{u} e_{w}-W e_{u} e_{v}+e_{u} e_{v} e_{w},
\end{aligned}
$$

where $e_{u}=U-u, e_{v}=V-v, e_{w}=W-w$.

Proof. This identity can be verified by direct computing.

For convenience, we define a Sobolev space:

$$
Y=C^{4}\left([0, T], W^{2, \infty}(D)\right) \cap C^{3}\left([0, T], W^{4, \infty}(D)\right) \cap C^{2}\left([0, T], W^{6, \infty}(D) \cap H_{0}^{1}(D)\right) .
$$

For simplicity, $\varphi \lesssim\left(\tau^{2}+h^{6}\right)$ denotes that there exists a constant $C$ independent of $\tau$ and $h$, such that $\varphi \leq C\left(\tau^{2}+h^{6}\right)$.

Theorem 3.4. Assume that, in the CGP equations (1.1a)-(1.1b), the potential functions $s_{1}(x, y)$, $s_{2}(x, y) \in C(D)$, and the exact solutions $W_{j k}^{n}=\left[U_{j k}^{n}, V_{j k}^{n}\right]^{T} \in Y \times Y$. Then there are two constants $\tau_{0}$ and $h_{0}$, for sufficiently small $\tau \in\left(0, \tau_{0}\right], h \in\left(0, h_{0}\right]$, we have the following local truncation error estimation for the HOC-AVF scheme (2.11a)-(2.11b)

$$
\left\|\varepsilon_{1}^{n}\right\| \lesssim\left(h^{6}+\tau^{2}\right), \quad\left\|\varepsilon_{2}^{n}\right\| \lesssim\left(h^{6}+\tau^{2}\right), \quad n \in T_{N}
$$


Proof. We define the point-wise local truncation error at $\left(x_{j}, y_{k}, t_{n}\right)$

$$
\begin{gathered}
\varepsilon_{1 j k}^{n}=i \delta_{t} U_{j k}^{n}+\frac{1}{2}\left(\mathcal{D}_{x x} \delta_{x}^{2}+\mathcal{D}_{y y} \delta_{y}^{2}\right) U_{j k}^{n+\frac{1}{2}}-s_{1 j k} U_{j k}^{n+\frac{1}{2}}+L_{h, z j, k} U_{j k}^{n+\frac{1}{2}}+\lambda V_{j k}^{n+\frac{1}{2}} \\
-\frac{\beta_{11}}{6}\left(\left|U_{j k}^{n}\right|^{2} U_{j k}^{n}+\left|U_{j k}^{n+1}\right|^{2} U_{j k}^{n+1}+4\left|U_{j k}^{n+\frac{1}{2}}\right|^{2} U_{j k}^{n+\frac{1}{2}}\right) \\
-\frac{\beta_{12}}{6}\left(\left|V_{j k}^{n}\right|^{2} U_{j k}^{n}+\left|V_{j k}^{n+1}\right|^{2} U_{j k}^{n+1}+4\left|V_{j k}^{n+\frac{1}{2}}\right|^{2} U_{j k}^{n+\frac{1}{2}}\right), \\
\varepsilon_{2 j k}^{n}=i \delta_{t} V_{j k}^{n}+\frac{1}{2}\left(\mathcal{D}_{x x} \delta_{x}^{2}+\mathcal{D}_{y y} \delta_{y}^{2}\right) V_{j k}^{n+\frac{1}{2}}-s_{2 j k} V_{j k}^{n+\frac{1}{2}}+L_{h, z j, k} V_{j k}^{n+\frac{1}{2}}+\lambda U_{j k}^{n+\frac{1}{2}} \\
-\frac{\beta_{21}}{6}\left(\left|U_{j k}^{n}\right|^{2} V_{j k}^{n}+\left|U_{j k}^{n+1}\right|^{2} V_{j k}^{n+1}+4\left|U_{j k}^{n+\frac{1}{2}}\right|^{2} V_{j k}^{n+\frac{1}{2}}\right) \\
-\frac{\beta_{22}}{6}\left(\left|V_{j k}^{n}\right|^{2} V_{j k}^{n}+\left|V_{j k}^{n+1}\right|^{2} V_{j k}^{n+1}+4\left|V_{j k}^{n+\frac{1}{2}}\right|^{2} V_{j k}^{n+\frac{1}{2}}\right) .
\end{gathered}
$$

Let

$$
\hat{U}=U\left(t_{n+\frac{1}{2}}\right), \quad \hat{U}_{t}=U_{t}\left(t_{n+\frac{1}{2}}\right), \quad \hat{V}=V\left(t_{n+\frac{1}{2}}\right), \quad \hat{V}_{t}=V_{t}\left(t_{n+\frac{1}{2}}\right),
$$

etc. by Taylor expansion, we have

$$
\begin{array}{ll}
U^{n}=\hat{U}-\frac{\tau}{2} \hat{U}_{t}+\frac{\tau^{2}}{8} \hat{U}_{t t}+\mathcal{O}\left(\tau^{3}\right), & V^{n+1}=\hat{V}+\frac{\tau}{2} \hat{V}_{t}+\frac{\tau^{2}}{8} \hat{V}_{t t}+\mathcal{O}\left(\tau^{3}\right), \\
U^{n+\frac{1}{2}}=\frac{1}{2}\left(U^{n}+U^{n+1}\right)=\hat{U}+\frac{\tau^{2}}{8} \hat{U}_{t t}+\mathcal{O}\left(\tau^{4}\right), & \delta_{t} V_{j k}^{n}=\hat{V}_{t}+\frac{\tau^{2}}{24} \hat{V}_{t t t}+\mathcal{O}\left(\tau^{4}\right) .
\end{array}
$$

It is obvious that we have

$$
\left|U^{n}\right|^{2}=|\hat{U}|^{2}-\frac{\tau}{2}\left(|\hat{U}|^{2}\right)_{t}+\mathcal{O}\left(\tau^{2}\right), \quad\left|V^{n+1}\right|^{2}=|\hat{V}|^{2}+\frac{\tau}{2}\left(|\hat{V}|^{2}\right)_{t}+\mathcal{O}\left(\tau^{2}\right),
$$

Thus, one has

$$
\left|U^{n}\right|^{2} V^{n}+\left|U^{n+1}\right|^{2} V^{n+1}=2|\hat{U}|^{2} \hat{V}+\mathcal{O}\left(\tau^{2}\right), \quad\left|U^{n+\frac{1}{2}}\right|^{2} V^{n+\frac{1}{2}}=|\hat{U}|^{2} \hat{V}+\mathcal{O}\left(\tau^{2}\right) .
$$

This implies that the local truncation errors from time in the nonlinear part in $\varepsilon_{1}{ }_{j k}^{n}, \varepsilon_{2}{ }_{j k}^{n}$ are $\mathcal{O}\left(\tau^{2}\right)$. It is clearly that the local truncation errors from time in the linear part in $\varepsilon_{1 j k}^{n}, \varepsilon_{2 j k}^{n}$ are $\mathcal{O}\left(\tau^{2}\right)$. Therefore, the total local truncation errors from time in $\varepsilon_{1 j k}^{n}, \varepsilon_{2 j k}^{n}$ are $\mathcal{O}\left(\tau^{2}\right)$, i.e., second order in time. In the following we are concerned with the local truncation error from space.

It can be observed from the definition of $A_{x}, A_{x x}, B_{x}, B_{x x}$ that 


$$
\left\{\begin{array}{l}
A_{x} U_{x j}=\frac{\partial U\left(x_{j}\right)}{\partial x}+\frac{1}{5} \frac{\partial^{3} U\left(x_{j}\right)}{\partial x^{3}} h_{x}^{2}+\frac{1}{60} \frac{\partial^{5} U\left(x_{j}\right)}{\partial x^{5}} h_{x}^{4}+\mathcal{O}\left(h_{x}^{6}\right), \\
B_{x} U_{j}=\frac{\partial U\left(x_{j}\right)}{\partial x}+\frac{1}{5} \frac{\partial^{3} U\left(x_{j}\right)}{\partial x^{3}} h_{x}^{2}+\frac{1}{60} \frac{\partial^{5} U\left(x_{j}\right)}{\partial x^{5}} h_{x}^{4}+\mathcal{O}\left(h_{x}^{6}\right), \\
A_{x x} U_{x x j}=\frac{\partial^{2} U\left(x_{j}\right)}{\partial x^{2}}+\frac{2}{15} \frac{\partial^{4} U\left(x_{j}\right)}{\partial x^{4}} h_{x}^{2}+\frac{1}{90} \frac{\partial^{6} U\left(x_{j}\right)}{\partial x^{6}} h_{x}^{4}+\mathcal{O}\left(h_{x}^{6}\right), \\
B_{x x} \delta_{x}^{2} U_{j}=\frac{\partial^{2} U\left(x_{j}\right)}{\partial x^{2}}+\frac{2}{15} \frac{\partial^{4} U\left(x_{j}\right)}{\partial x^{4}} h_{x}^{2}+\frac{1}{90} \frac{\partial^{6} U\left(x_{j}\right)}{\partial x^{6}} h_{x}^{4}+\mathcal{O}\left(h_{x}^{6}\right) .
\end{array}\right.
$$

To get the local truncation errors in the $x$ and $y$ directions, we consider them one by one. First, we derive it in the $x$ direction. To the purpose, we rewrite (1.1a) and (3.13a) as

$$
\begin{aligned}
& U_{x x}+2 \Omega i y U_{x}=-2 i U_{t}-U_{y y}+2 \Omega i x U_{y}+2\left[s_{1} U+\left(\beta_{11}|U|^{2}+\beta_{12}|V|^{2}\right) U-\lambda V\right] \\
& \triangleq F(x, y) \text {, } \\
& A_{x} B_{x x} \delta_{x}^{2} U_{j k}^{n+\frac{1}{2}}+2 i \Omega y_{k} A_{x x} B_{x} U_{j k}^{n+\frac{1}{2}} \\
& =A_{x x} A_{x}\left\{2 \varepsilon_{1 j k}^{n}-2 i \delta_{t} U_{j k}^{n}-\mathcal{D}_{y y} \delta_{y}^{2} U_{j k}^{n+\frac{1}{2}}+2 s_{1 j k} U_{j k}^{n+\frac{1}{2}}+2 i \Omega x_{j} \mathcal{D}_{y} U_{j k}^{n+\frac{1}{2}}\right. \\
& -2 \lambda V_{j k}^{n+\frac{1}{2}}+\frac{2 \beta_{11}}{6}\left(\left|U_{j k}^{n}\right|^{2} U_{j k}^{n}+\left|U_{j k}^{n+1}\right|^{2} U_{j k}^{n+1}+4\left|U_{j k}^{n+\frac{1}{2}}\right|^{2} U_{j k}^{n+\frac{1}{2}}\right) \\
& \left.+\frac{2 \beta_{12}}{6}\left(\left|V_{j k}^{n}\right|^{2} U_{j k}^{n}+\left|V_{j k}^{n+1}\right|^{2} U_{j k}^{n+1}+4\left|V_{j k}^{n+\frac{1}{2}}\right|^{2} U_{j k}^{n+\frac{1}{2}}\right)\right\} \\
& \triangleq A_{x x} A_{x}\left[2 \varepsilon_{1 j k}^{n}+F_{j k}^{n+\frac{1}{2}}\right] \text {. }
\end{aligned}
$$

From Eq. (3.15a), one has

$$
\frac{\partial^{4} U}{\partial x^{4}}+2 \Omega i y \frac{\partial^{3} U}{\partial x^{3}}-\frac{\partial^{2} F}{\partial x^{2}}=0, \quad \frac{\partial^{6} U}{\partial x^{6}}+2 \Omega i y \frac{\partial^{5} U}{\partial x^{5}}-\frac{\partial^{4} F}{\partial x^{4}}=0 .
$$

Inserting Eqs. (3.14) into Eq. (3.15b), it yields

$$
\begin{aligned}
& 2 A_{x} A_{x x} \varepsilon_{1 j k}^{n}=A_{x} B_{x x} \delta_{x}^{2} U_{j k}+2 i \Omega y_{k} A_{x x} B_{x} U_{j k}-A_{x x} A_{x} F_{j k} \\
=A_{x} & {\left[\frac{\partial^{2} U}{\partial x^{2}}+\frac{2}{15} \frac{\partial^{4} U}{\partial x^{4}} h_{x}^{2}+\frac{1}{90} \frac{\partial^{6} U}{\partial x^{6}} h_{x}^{4}\right]+2 \Omega i y_{k} A_{x x}\left[\frac{\partial U}{\partial x}+\frac{1}{5} \frac{\partial^{3} U}{\partial x^{3}} h_{x}^{2}+\frac{1}{60} \frac{\partial^{5} U}{\partial x^{5}} h_{x}^{4}\right] } \\
& -A_{x x}\left[F_{j k}+\frac{1}{5} \frac{\partial^{2} F}{\partial x^{2}} h_{x}^{2}+\frac{1}{60} \frac{\partial^{4} F}{\partial x^{4}} h_{x}^{4}\right]+2 \varepsilon_{1 j k}^{n}+\mathcal{O}\left(h_{x}^{6}\right) \\
= & {\left[\frac{\partial^{2} U}{\partial x^{2}}+\frac{1}{5} \frac{\partial^{4} U}{\partial x^{4}} h_{x}^{2}+\frac{1}{60} \frac{\partial^{6} U}{\partial x^{6}} h_{x}^{4}\right]+\frac{2}{15}\left[\frac{\partial^{4} U}{\partial x^{4}}+\frac{1}{5} \frac{\partial^{6} U}{\partial x^{6}} h_{x}^{2}\right] h_{x}^{2}+\frac{1}{90} \frac{\partial^{6} U}{\partial x^{6}} h_{x}^{4}+2 \Omega i y_{k} } \\
& \times\left[\left(\frac{\partial U}{\partial x}+\frac{2}{15} \frac{\partial^{3} U}{\partial x^{3}} h_{x}^{2}+\frac{1}{90} \frac{\partial^{5} U}{\partial x^{5}} h_{x}^{4}\right)+\frac{1}{5}\left(\frac{\partial^{3} U}{\partial x^{3}}+\frac{2}{15} \frac{\partial^{5} U}{\partial x^{5}} h_{x}^{2}\right) h_{x}^{2}+\frac{1}{60} \frac{\partial^{5} U}{\partial x^{5}} h_{x}^{4}\right]
\end{aligned}
$$




$$
\begin{aligned}
& -\left[F+\frac{2}{15} \frac{\partial^{2} F}{\partial x^{2}} h_{x}^{2}+\frac{1}{90} \frac{\partial^{4} F}{\partial x^{4}} h_{x}^{4}\right]-\frac{1}{5}\left[\frac{\partial^{2} F}{\partial x^{2}}+\frac{2}{15} \frac{\partial^{4} F}{\partial x^{4}} h_{x}^{2}\right] h_{x}^{2}-\frac{1}{60} \frac{\partial^{4} F}{\partial x^{4}} h_{x}^{4}+\mathcal{O}\left(h_{x}^{6}\right) \\
= & \left(\frac{\partial^{2} U}{\partial x^{2}}+2 \Omega i y_{k} \frac{\partial U}{\partial x}-F\right)+\frac{1}{3}\left(\frac{\partial^{4} U}{\partial x^{4}}+2 \Omega i y_{k} \frac{\partial^{3} U}{\partial x^{3}}-\frac{\partial^{2} F}{\partial x^{2}}\right) h_{x}^{2} \\
& \quad+\frac{49}{900}\left(\frac{\partial^{6} U}{\partial x^{6}}+2 \Omega i y_{k} \frac{\partial^{5} U}{\partial x^{5}}-\frac{\partial^{4} F}{\partial x^{4}}\right) h_{x}^{4}+\mathcal{O}\left(h_{x}^{6}\right) \\
=\mathcal{O}\left(h_{x}^{6}\right) . &
\end{aligned}
$$

In the last equality we have used the equalities (3.16). For simplicity and convenient, we also neglected all the time index $n+\frac{1}{2}$ and spatial index $(j, k)$ without confusion. Therefore, the truncation error $\varepsilon_{1 j k}^{n}$ in the $x$-direction is of sixth order due to the boundedness of $A_{x}, A_{x x}$. Therefore, the HOC-AVF scheme (2.11a)-(2.11b) is of sixth order in the $x$ direction. With a similar procedure, one can find that it is of sixth order in $y$ direction. We can derive that $\varepsilon_{2 j k}^{n}$ is also of sixth order in space. In summary, the estimation (3.12) is true. This completes the proof.

Define the global point-wise error functions $e_{1}{ }_{j k}^{n}, e_{2 j k}^{n} \in X_{p}$ as

$$
e_{1 j k}^{n}=U_{j k}^{n}-u_{j k}^{n}, \quad e_{2 j k}^{n}=V_{j k}^{n}-v_{j k}^{n}, \quad(j, k) \in S_{p}, \quad n \in T_{N} .
$$

To get the global error estimation, we need the follows lemmas:

Lemma 3.3 ([32,36]). For any grid function $u \in X_{p}$, there is

$$
\|u\|_{4}^{4} \leq\|u\|^{2}\left(2\left\|\nabla_{h} u\right\|+\|u\| / l\right)^{2}, \text { where } l=\min \left\{x_{r}-x_{l}, y_{r}-y_{l}\right\} .
$$

Lemma 3.4. For any grid function $u \in X_{p}$, the two semi-norms $|u|_{1}$ and $\left.\widetilde{\mid u}\right|_{1}$ of $u$ defined previously are equivalent, that is, there exists two positive constants $C_{1}=1$ and $C_{2}=2 \sqrt{3 / 7}$, such that

$$
C_{1}\left\|\nabla_{h} u\right\| \leq \widetilde{\left\|\nabla_{h} u\right\|} \leq C_{2}\left\|\nabla_{h} u\right\| .
$$

Proof. Reminding that $D_{x x}=A_{x x}^{-1} B_{x x}, D_{y y}=A_{y y}^{-1} B_{y y}$ and that $A_{x x}, A_{y y}, B_{x x}, B_{y y}$ are circlant matrices [32], their eigenvalues are in the form

$$
\begin{array}{ll}
\lambda_{A_{x x j}}=\frac{1}{15}\left(11+4 \cos \theta_{x j}\right), & \lambda_{B_{x x j}}=\frac{1}{20}\left(18+2 \cos \theta_{x j}\right), \\
\lambda_{A_{y y k}}=\frac{1}{15}\left(11+4 \cos \theta_{y_{k}}\right), & \lambda_{B_{y y}}=\frac{1}{20}\left(18+2 \cos \theta_{y_{k}}\right),
\end{array}
$$

where $\theta_{x j}=2 j \pi / J$ and $\theta_{y_{k}}=2 k \pi / K$. Therefore, the eigenvalues of $D_{x x}$ and $D_{y y}$ are

$$
\begin{aligned}
& \lambda_{D_{x x j}}=\frac{\left(2 \cos \theta_{x j}+18\right) / 20}{\left(4 \cos \theta_{x j}+11\right) / 15}=\frac{3}{2} \frac{\cos \theta_{x j}+9}{4 \cos \theta_{x j}+11}, \quad j=1, \cdots, J, \\
& \lambda_{D_{y y k}}=\frac{\left(2 \cos \theta_{y_{k}}+18\right) / 20}{\left(4 \cos \theta_{y_{k}}+11\right) / 15}=\frac{3}{2} \frac{\cos \theta_{y_{k}}+9}{4 \cos \theta_{y_{k}}+11}, \quad k=1, \cdots, K .
\end{aligned}
$$


It is clear that $1 \leq \lambda_{D_{x x} j} \leq 12 / 7$ and $1 \leq \lambda_{D_{y y}} \leq 12 / 7$ for all $j, k$. Thus, the spectral radius $\rho\left(D_{x x}\right) \leq 12 / 7$ and $\rho\left(D_{y y}\right) \leq 12 / 7$, and consequently

$$
\begin{aligned}
\left\|\nabla_{h} u\right\|^{2} & \leq-\left\langle D_{x x} \delta_{x}^{2} u, u\right\rangle-\left\langle D_{y y} \delta_{y}^{2} u, u\right\rangle={\widetilde{\nabla_{h} u \|^{2}}}^{2} \\
& =\left\langle D_{x x} \delta_{x}^{+} u, \delta_{x}^{+} u\right\rangle+\left\langle D_{y y} \delta_{y}^{+} u, \delta_{y}^{+} u\right\rangle \\
& =\lambda_{D_{x x}}\left\|\delta_{x}^{+} u\right\|^{2}+\lambda_{D_{y y}}\left\|\delta_{y}^{+} u\right\|^{2} \\
& \leq \frac{12}{7}\left\|\nabla_{h} u\right\|^{2} .
\end{aligned}
$$

This suggests that the two semi-norms are equivalent. The proof is completed.

For the numerical solutions of the HOC-AVF scheme (2.11a)-(2.11b), we have the following error estimations.

Theorem 3.5. Let the assumptions of Theorem 3.4 hold, moreover, assume that the system lacks of rotation $(\Omega=0)$ and Josephson junction $(\lambda=0)$, and the interaction constant matrix $\beta$ is a non-negative or positive semi-definite matrix, then we have the following global error estimation for the HOC-AVF scheme (2.11a)-(2.11b)

$$
\left\|e_{1}^{n}\right\| \lesssim\left(h^{6}+\tau^{2}\right), \quad\left\|e_{2}^{n}\right\| \lesssim\left(h^{6}+\tau^{2}\right), \quad n \in T_{N}
$$

Proof. Subtracting Eqs. (3.13a)-(3.13b) from Eqs. (2.11a)-(2.11b) with the assumption $\Omega=$ $\lambda=0$, one can obtain the error equations

$$
\begin{aligned}
& \varepsilon_{1 j k}^{n}=i \delta_{t} e_{1 j k}^{n}+\frac{1}{2}\left(\mathcal{D}_{x x} \delta_{x}^{2}+\mathcal{D}_{y y} \delta_{y}^{2}\right) e_{1 j k}^{n+\frac{1}{2}}-s_{1 j k} e_{1 j k}^{n+\frac{1}{2}}-G_{1 j k}^{n} \\
& \varepsilon_{2 j k}^{n}=i \delta_{t} e_{2 j k}^{n}+\frac{1}{2}\left(\mathcal{D}_{x x} \delta_{x}^{2}+\mathcal{D}_{y y} \delta_{y}^{2}\right) e_{2 j k}^{n+\frac{1}{2}}-s_{2 j k} e_{2 j k}^{n+\frac{1}{2}}-G_{2 j k}^{n},
\end{aligned}
$$

or the vector form

$$
\varepsilon^{n}=i \delta_{t} e^{n}+\frac{1}{2}\left(\mathcal{D}_{x x} \delta_{x}^{2}+\mathcal{D}_{y y} \delta_{y}^{2}\right) e^{n+\frac{1}{2}}-s \cdot e^{n+\frac{1}{2}}-G^{n},
$$

where

$$
\begin{aligned}
G_{1 j k}^{n}=\frac{\beta_{11}}{6} & {\left[\left(\left|U_{j k}^{n}\right|^{2} U_{j k}^{n}-\left|u_{j k}^{n}\right|^{2} u_{j k}^{n}\right)+\left(\left|u_{j k}^{n+1}\right|^{2} U_{j k}^{n+1}-\left|u_{j k}^{n+1}\right|^{2} u_{j k}^{n+1}\right)\right.} \\
& \left.+4\left(\left|u_{j k}^{n+\frac{1}{2}}\right|^{2} U_{j k}^{n+\frac{1}{2}}-\left|u_{j k}^{n+\frac{1}{2}}\right|^{2} u_{j k}^{n+\frac{1}{2}}\right)\right]+\frac{\beta_{12}}{6}\left[\left(\left|V_{j k}^{n}\right|^{2} u_{j k}^{n}-\left|v_{j k}^{n}\right|^{2} u_{j k}^{n}\right)\right. \\
& \left.+\left(\left|V_{j k}^{n+1}\right|^{2} U_{j k}^{n+1}-\left|v_{j k}^{n+1}\right|^{2} u_{j k}^{n+1}\right)+4\left(\left|V_{j k}^{n+\frac{1}{2}}\right|^{2} U_{j k}^{n+\frac{1}{2}}-\left|v_{j k}^{n+\frac{1}{2}}\right|^{2} u_{j k}^{n+\frac{1}{2}}\right)\right]
\end{aligned}
$$




$$
\begin{aligned}
& =\frac{\beta_{11}}{6}\left\{\left[\left(U_{j k}^{n}\right)^{2} \overline{e_{1 j k}^{n}}+\left(U_{j k}^{n+1}\right)^{2} \overline{e_{1 j k}^{n+1}}\right]+2\left(\left|u_{j k}^{n}\right|^{2} e_{1 j k}^{n}+\left|U_{j k}^{n+1}\right|^{2} e_{1 j k}^{n+1}\right)\right. \\
& -2\left(U_{j k}^{n}\left|e_{1 j k}^{n}\right|^{2}+U_{j k}^{n+1}\left|e_{1 j k}^{n+1}\right|^{2}\right)-\left(\overline{U_{j k}^{n}}\left(e_{1 j k}^{n}\right)^{2}+\overline{U_{j k}^{n+1}}\left(e_{1 j k}^{n+1}\right)^{2}\right) \\
& \left.+\left(\left|e_{1 j k}^{n}\right|^{2} e_{1 j k}^{n}+\left|e_{1 j k}^{n+1}\right|^{2} e_{1 j k}^{n+1}\right)+4\left[\left(U_{j k}^{n+\frac{1}{2}}\right)^{2 \overline{e_{1}}} e_{j k}^{n+\frac{1}{2}}+U_{j k}^{n+\frac{1}{2}}\left|e_{1_{j k}^{n+\frac{1}{2}}}\right|^{2}\right]\right\} \\
& +\frac{\beta_{12}}{6}\left\{2\left[\mathcal{R}\left(V_{j k}^{n} \overline{e_{2 j k}^{n}}\right)\left(U_{j k}^{n}-e_{1 j k}^{n}\right)+\mathcal{R}\left(V_{j k}^{n+1} \overline{e_{2 j k}^{n+1}}\right)\left(U_{j k}^{n+1}-e_{1 j k}^{n+1}\right)\right]\right. \\
& +\left(\left|V_{j k}^{n}\right|^{2} e_{1 j k}^{n}+\left|V_{j k}^{n+1}\right|^{2} e_{1 j k}^{n+1}\right)-\left(U_{j k}^{n}\left|e_{2 j k}^{n}\right|^{2}+U_{j k}^{n+1}\left|e_{2 j k}^{n+1}\right|^{2}\right) \\
& +\left(\left|e_{2 j k}^{n}\right|^{2} e_{1 j k}^{n}+\left|e_{2 j k}^{n+1}\right|^{2} e_{1 j k}^{n+1}\right)+4\left[2 U_{j k}^{n+\frac{1}{2}} \mathcal{R}\left(V_{j k}^{n+\frac{1}{2}} \overline{e_{2 j k}^{n+\frac{1}{2}}}\right)\right. \\
& \left.\left.+\left[\left|V_{j k}^{n+\frac{1}{2}}\right|^{2}-2 \mathcal{R}\left(V_{j k}^{n+\frac{1}{2}} \overline{e_{2 j k}^{n+\frac{1}{2}}}\right)\right] e_{1_{j k}^{n+\frac{1}{2}}}-\left[U_{j k}^{n+\frac{1}{2}}-e_{1_{j k}^{n+\frac{1}{2}}}^{n}\right]\left|e_{2 j k}^{n+\frac{1}{2}}\right|^{2}\right]\right\} \\
& +\frac{4 \beta_{11}}{6}\left\{\left(2\left|U_{j k}^{n+\frac{1}{2}}\right|^{2}+\left|e_{1 j k}^{n+\frac{1}{2}}\right|^{2}\right)-\left(U_{j k}^{n+1} \overline{e_{1 j k}^{n+\frac{1}{2}}}+\overline{U_{j k}^{n+1}} e_{1 j k}^{n+\frac{1}{2}}\right)\right\} e_{1 j k}^{n+\frac{1}{2}} \\
& G_{2 j k}^{n}=\frac{\beta_{21}}{6}\left[\left(\left|U_{j k}^{n}\right|^{2} V_{j k}^{n}-\left|u_{j k}^{n}\right|^{2} v_{j k}^{n}\right)+\left(\left|U_{j k}^{n+1}\right|^{2} V_{j k}^{n+1}-\left|u_{j k}^{n+1}\right|^{2} v_{j k}^{n+1}\right)\right. \\
& \left.+4\left(\left|U_{j k}^{n+\frac{1}{2}}\right|^{2} V_{j k}^{n+\frac{1}{2}}-\left|u_{j k}^{n+\frac{1}{2}}\right|^{2} v_{j k}^{n+\frac{1}{2}}\right)\right]+\frac{\beta_{22}}{6}\left[\left(\left|V_{j k}^{n}\right|^{2} V_{j k}^{n}-\left|v_{j k}^{n}\right|^{2} v_{j k}^{n}\right)\right. \\
& \left.+\left(\left|V_{j k}^{n+1}\right|^{2} V_{j k}^{n+1}-\left|v_{j k}^{n+1}\right|^{2} v_{j k}^{n+1}\right)+4\left(\left|V_{j k}^{n+\frac{1}{2}}\right|^{2} V_{j k}^{n+\frac{1}{2}}-\left|v_{j k}^{n+\frac{1}{2}}\right|^{2} v_{j k}^{n+\frac{1}{2}}\right)\right] \\
& =\frac{\beta_{21}}{6}\left\{2\left[\mathcal{R}\left(U_{j k}^{n} \overline{e_{1 j k}^{n}}\right)\left(V_{j k}^{n}-e_{2 j k}^{n}\right)+\mathcal{R}\left(U_{j k}^{n+1} \overline{e_{1 j k}^{n+1}}\right)\left(V_{j k}^{n+1}-e_{2 j k}^{n+1}\right)\right]\right. \\
& +\left(\left|U_{j k}^{n}\right|^{2} e_{2 j k}^{n}+\left|U_{j k}^{n+1}\right|^{2} e_{2 j k}^{n+1}\right)-\left(V_{j k}^{n}\left|e_{1 j k}^{n}\right|^{2}+V_{j k}^{n+1}\left|e_{1 j k}^{n+1}\right|^{2}\right) \\
& +\left(\left|e_{1 j k}^{n}\right|^{2} e_{2 j k}^{n}+\left|e_{1 j k}^{n+1}\right|^{2} e_{2 j k}^{n+1}\right)+4\left[2 V_{j k}^{n+\frac{1}{2}} \mathcal{R}\left(U_{j k}^{n+\frac{1}{2}} \overline{e_{1 j k}^{n+\frac{1}{2}}}\right)\right. \\
& \left.\left.+\left[\left|U_{j k}^{n+\frac{1}{2}}\right|^{2}-2 \mathcal{R}\left(U_{j k}^{n+\frac{1}{2}} \overline{e_{1 j k}^{n+\frac{1}{2}}}\right)\right] e_{2 j k}^{n+\frac{1}{2}}-\left(V_{j k}^{n+\frac{1}{2}}-e_{2 j k}^{n+\frac{1}{2}}\right)\left|e_{1_{j k}}^{n+\frac{1}{2}}\right|^{2}\right]\right\} \\
& +\frac{\beta_{22}}{6}\left\{\left[\left(V_{j k}^{n}\right)^{2} \overline{e_{2 j k}^{n}}+\left(V_{j k}^{n+1}\right)^{2} \overline{e_{2 j k}^{n+1}}\right]+2\left(\left|V_{j k}^{n}\right|^{2} e_{2 j k}^{n}+\left|V_{j k}^{n+1}\right|^{2} e_{2 j k}^{n+1}\right)\right. \\
& -2\left(V_{j k}^{n}\left|e_{2 j k}^{n}\right|^{2}+V_{j k}^{n+1}\left|e_{2 j k}^{n+1}\right|^{2}\right)-\left(\overline{V_{j k}^{n}}\left(e_{2 j k}^{n}\right)^{2}+\overline{V_{j k}^{n+1}}\left(e_{2 j k}^{n+1}\right)^{2}\right)
\end{aligned}
$$




$$
\begin{aligned}
& \left.+\left(\left|e_{2 j k}^{n}\right|^{2} e_{2 j k}^{n}+\left|e_{2 j k}^{n+1}\right|^{2} e_{2 j k}^{n+1}\right)+4\left[\left(V_{j k}^{n+\frac{1}{2}}\right)^{2 \overline{e_{2}}{ }_{2 j k}^{n+\frac{1}{2}}}+V_{j k}^{n+\frac{1}{2}}\left|e_{2 j k}^{n+\frac{1}{2}}\right|^{2}\right]\right\} \\
& +\frac{4 \beta_{22}}{6}\left\{\left(2\left|V_{j k}^{n+\frac{1}{2}}\right|^{2}+\left|e_{2 j k}^{n+\frac{1}{2}}\right|^{2}\right)-\left(V_{j k}^{n+1} \overline{e_{2 j k}^{n+\frac{1}{2}}}+\overline{V_{j k}^{n+1}} e_{2 j k}^{n+\frac{1}{2}}\right)\right\} e_{2 j k}^{n+\frac{1}{2}} \\
& \triangleq G_{21}+G_{22},
\end{aligned}
$$

where $G_{m 1}$ is the first two parts in $G_{m}$, and $G_{m 2}$ is the last part in $G_{m}, m=1,2$.

To estimate the global error (3.21), we list some prior estimations:

From the discrete energy conservation law (3.1) and the exact solution $W=[U, V]^{T} \in$ $Y \times Y$, we have

$$
\begin{aligned}
& \left\|u^{n}\right\|_{4}^{4} \leq C, \quad\left\|v^{n}\right\|_{4}^{4} \leq C, \quad\left\|\widetilde{\nabla_{h} u^{n}}\right\| \leq C, \quad\left\|\widetilde{\nabla_{h} V^{n}}\right\| \leq C, \\
& \left\|U^{n}\right\|_{\infty}=\max _{j, k}\left|U_{j k}^{n}\right| \leq C, \quad\left\|V^{n}\right\|_{\infty}=\max _{j, k}\left|V_{j k}^{n}\right| \leq C .
\end{aligned}
$$

From these prior estimations, it derives that

$$
\left\|e^{n}\right\|_{4}^{4}=\left\|e_{1}^{n}\right\|_{4}^{4}+\left\|e_{2}^{n}\right\|_{4}^{4} \leq\left(\left\|u^{n}\right\|_{4}^{4}+\left\|U^{n}\right\|_{4}^{4}\right)+\left(\left\|v^{n}\right\|_{4}^{4}+\left\|V^{n}\right\|_{4}^{4}\right) \leq C .
$$

Taking inner product of Eq. (3.23) with $e^{n+\frac{1}{2}}$, then taking the imaginary part, we have

$$
\begin{aligned}
\frac{\left\|e^{n+1}\right\|^{2}-\left\|e^{n}\right\|^{2}}{2 \tau} & =\operatorname{Im}\left(\left\langle\varepsilon^{n}, e^{n+\frac{1}{2}}\right\rangle\right)+\operatorname{Im}\left(\left\langle G^{n}, e^{n+\frac{1}{2}}\right\rangle\right) \\
& =\operatorname{Im}\left(\left\langle\varepsilon^{n}, e^{n+\frac{1}{2}}\right\rangle\right)+\operatorname{Im}\left(\left\langle G_{11}^{n}, e_{1}^{n+\frac{1}{2}}\right\rangle+\left\langle G_{21}^{n}, e_{2}^{n+\frac{1}{2}}\right\rangle\right) .
\end{aligned}
$$

Here we have used the fact that $\left\langle G_{12}^{n}, e_{1}^{n+\frac{1}{2}}\right\rangle$ and $\left\langle G_{22}^{n}, e_{2}^{n+\frac{1}{2}}\right\rangle$ are real numbers. Consequently, by the prior estimates (3.24), (3.25), the Cauchy-Schwartz inequality and Young inequality, etc, one has

$$
\begin{aligned}
& \operatorname{Im}\left(\left\langle G^{n}, e^{n+\frac{1}{2}}\right\rangle\right) \leq\left|\left\langle G_{11}^{n}, e_{1}^{n+\frac{1}{2}}\right\rangle\right|+\left|\left\langle G_{21}^{n}, e_{2}^{n+\frac{1}{2}}\right\rangle\right| \\
& \lesssim h_{x} h_{y} \sum_{j, k}\left\{\left[\left|e_{1 j k}^{n}\right|+\left|e_{1 j k}^{n+1}\right|+\left|e_{2 j k}^{n}\right|+\left|e_{2 j k}^{n+1}\right|+\left|e_{1 j k}^{n+\frac{1}{2}}\right|+\left|e_{2 j k}^{n+\frac{1}{2}}\right|\right]+\left[\left|e_{1 j k}^{n}\right|^{2}\right.\right. \\
& +\left|e_{1 j k}^{n+1}\right|^{2}+\left|e_{2 j k}^{n}\right|^{2}+\left|e_{2 j k}^{n+1}\right|^{2}+\left|e_{1 j k}^{n+\frac{1}{2}}\right|^{2}+\left|e_{2 j k}^{n+\frac{1}{2}}\right|^{2}+\left|e_{1 j k}^{n} e_{2 j k}^{n}\right|+\left|e_{1_{j k}}^{n+1} e_{2_{j k}^{n+1}}^{n+1}\right| \\
& \left.+\left|e_{1 j k}^{n+\frac{1}{2}} e_{2 j k}^{n+\frac{1}{2}}\right|\right]+\left[\left|e_{1 j k}^{n}\right|^{3}+\left|e_{1 j k}^{n+1}\right|^{3}+\left|e_{2 j k}^{n}\right|^{3}+\left|e_{2 j k}^{n+1}\right|^{3}+\left|e_{1 j k}^{n}\right|\left|e_{2 j k}^{n}\right|^{2}\right. \\
& +\left|e_{1_{j k}}^{n+1}\right|\left|e_{2 j k}^{n+1}\right|^{2}+\left|e_{1 j k}^{n}\right|^{2}\left|e_{2 j k}^{n}\right|+\left|e_{1 j k}^{n+1}\right|^{2}\left|e_{2 j k}^{n+1}\right|+\left|e_{1 j k}^{n+\frac{1}{2}}\right|^{2}\left|e_{2 j k}^{n+\frac{1}{2}}\right|^{n} \\
& \left.\left.+\left|e_{1 j k}^{n+\frac{1}{2}}\right|\left|e_{2 j k}^{n+\frac{1}{2}}\right|^{2}\right]\right\} \times\left[\left|e_{1_{j k}}^{n+\frac{1}{2}}\right|+\left|e_{2 j k}^{n+\frac{1}{2}}\right|\right]
\end{aligned}
$$




$$
\begin{aligned}
& \lesssim\left[1+\left\|e_{1}^{n}\right\|_{4}^{4}+\left\|e_{2}^{n}\right\|_{4}^{4}+\left\|e_{1}^{n+1}\right\|_{4}^{4}+\left\|e_{2}^{n+1}\right\|_{4}^{4}\right]\left[\left\|\left(e_{1}^{n}, e_{2}^{n}\right)\right\|^{2}+\left\|\left(e_{1}^{n+1}, e_{2}^{n+1}\right)\right\|^{2}\right], \\
& \left|\operatorname{Im}\left(\left\langle\varepsilon^{n}, e^{n+\frac{1}{2}}\right\rangle\right)\right| \leq\left\|\left(\varepsilon_{1}^{n}, \varepsilon_{2}^{n}\right)\right\|^{2}+\left\|\left(e_{1}^{n}, e_{2}^{n}\right)\right\|^{2}+\left\|\left(e_{1}^{n+1}, e_{2}^{n+1}\right)\right\|^{2} .
\end{aligned}
$$

Thus, from estimates (3.27a) and (3.27b) and Eq. (3.26) one can obtain

$$
\begin{aligned}
\frac{\left\|e^{n+1}\right\|^{2}-\left\|e^{n}\right\|^{2}}{2 \tau} & =\operatorname{Im}\left(\left\langle\varepsilon^{n}, e^{n+\frac{1}{2}}\right\rangle\right)+\operatorname{Im}\left(\left\langle G^{n}, e^{n+\frac{1}{2}}\right\rangle\right) \leq\left|\left\langle\varepsilon^{n}, e^{n+\frac{1}{2}}\right\rangle\right|+\left|\left\langle G^{n}, e^{n+\frac{1}{2}}\right\rangle\right| \\
& \leq C\left(\left\|e^{n}\right\|^{2}+\left\|e^{n+1}\right\|^{2}\right)+C\left\|\varepsilon^{n}\right\|^{2} .
\end{aligned}
$$

By virtue of Lemma 3.1, we can obtain that the estimation (3.21) is correct. This completes the proof.

\section{Numerical experiments}

In this section, we investigate the HOC-AVF scheme (2.11a)-(2.11b) numerically to the CGP equations (1.1a)-(1.1b) with/without rotation. In all the examples, we choose the harmonic potential functions

$$
s_{1}(x, y)=s_{2}(x, y)=\frac{1}{2}\left(x^{2}+y^{2}\right),
$$

and in the first three examples, the initial data are Gaussian pulses

$$
u_{0}(x, y)=\frac{1}{\sqrt{2 \pi}} \exp \left(-\frac{x^{2}+y^{2}}{2}\right), \quad v_{0}(x, y)=\frac{\sqrt[4]{1.5}}{\sqrt{2 \pi}} \exp \left(-\frac{x^{2}+1.5 y^{2}}{2}\right) .
$$

Example 4.1 (Accuracy and energy-preserving test). We solve the CGP equations (1.1a)(1.1b) with parameters $\lambda=2, \Omega=0.6, \beta_{11}=\beta_{22}=10, \beta_{12}=\beta_{21}=7$ by the HOC-AVF scheme (2.11a)-(2.11b).

In this simulation, we choose a sufficiently large spatial domain $D=[-10,10]^{2}$ such that the solution is always almost equal to zero near the boundary points to avoid alias errors. First, we are interested in verifying the temporal and the spatial convergence rates. For comparison, the "exact" solution $\left[U_{j k}^{n}, V_{j k}^{n}\right]^{T}$ is obtained with very fine meshes, e.g., $h=\frac{20}{320}, \tau=\frac{1}{100000}$. To make the test more accurate, we stop the simulation at $t=1$. The point-wise errors are computed by $e\left(u_{j k}^{n}\right)=u_{j k}^{n}-U_{j k}^{n}, e\left(v_{j k}^{n}\right)=v_{j k}^{n}-V_{j k}^{n}$. The convergence rate is computed by

$$
\text { rate }=\frac{\ln \left(\left\|e\left(u^{n}\left(h_{1}\right)\right)\right\|_{p} /\left\|e\left(u^{n}\left(h_{2}\right)\right)\right\|_{p}\right)}{\ln \left(h_{1} / h_{2}\right)},
$$

where $e\left(u^{n}\left(h_{1}\right)\right)$ is the numerical error of $u_{j k}^{n}$ at $t_{n}$ obtained under mesh size $h_{1}$, and so on. We take $p=2$ and $p=\infty$ in the tests. 
Table 1: Verification of temporal convergence rate with $h=\frac{20}{320}$.

\begin{tabular}{||c|c|c|c|c|c|c|c|c||}
\hline$\tau$ & $\left\|e\left(u^{n}\right)\right\|_{\infty}$ & rate & $\left\|e\left(v^{n}\right)\right\|_{\infty}$ & rate & $\left\|e\left(u^{n}\right)\right\|_{2}$ & rate & $\left\|e\left(v^{n}\right)\right\|_{2}$ & rate \\
\hline$\frac{1}{4000}$ & $4.150 e-8$ & - & $1.667 e-8$ & - & $2.254 e-7$ & - & $1.212 e-7$ & - \\
\hline$\frac{1}{5000}$ & $2.653 e-8$ & 2.00 & $1.006 e-8$ & 2.00 & $1.441 e-7$ & 2.00 & $7.753 e-8$ & 2.00 \\
\hline$\frac{1}{8000}$ & $1.032 e-8$ & 2.00 & $4.148 e-9$ & 2.00 & $5.608 e-8$ & 2.00 & $3.017 e-8$ & 2.00 \\
\hline
\end{tabular}

Table 2: Verification of spatial convergence order with $\tau=\frac{1}{100000}$.

\begin{tabular}{||c|c|c|c|c|c|c|c|c||}
\hline$h$ & $\left\|e\left(u^{n}\right)\right\|_{\infty}$ & rate & $\left\|e\left(v^{n}\right)\right\|_{\infty}$ & rate & $\left\|e\left(u^{n}\right)\right\|_{2}$ & rate & $\left\|e\left(v^{n}\right)\right\|_{2}$ & rate \\
\hline$\frac{20}{40}$ & $1.897 e-3$ & - & $1.223 e-3$ & - & $1.858 e-3$ & - & $1.322 e-3$ & - \\
\hline$\frac{20}{80}$ & $2.447 e-5$ & 6.28 & $1.570 e-5$ & 6.28 & $5.298 e-5$ & 5.13 & $3.700 e-5$ & 5.16 \\
\hline$\frac{20}{160}$ & $4.159 e-7$ & 5.88 & $3.051 e-7$ & 5.68 & $2.042 e-6$ & 4.70 & $1.550 e-6$ & 4.58 \\
\hline
\end{tabular}

To get the temporal convergence rate, we fix a fine mesh $h=\frac{20}{320}$. Table 1 shows the spatial errors in the $l^{\infty}$ and $l^{2}$ norms and the corresponding convergence rate of the HOCAVF scheme (2.11a)-(2.11b) with different $\tau$. Table 2 displays similar results with fixed small time step $\tau=\frac{1}{100000}$ and different $h$.

From Tables 1-2, we can observe that the HOC-AVF scheme (2.11a)-(2.11b) is of second order in time and of sixth order in space, which verifies the theoretical analysis.

Then we simulate the above problem with the step size $h_{x}=h_{y}=\frac{20}{160}$ and $\tau=0.001$ in the time interval $[0,30]$. In this experiment, we are interested in the dynamic behavior of the wave function $\left|u_{j k}^{n}\right|^{2}$ and $\left|v_{j k}^{n}\right|^{2}$. Certainly, we will also pay attention to evolution of energy $\mathcal{H}^{n}-\mathcal{H}^{0}$ with time. We also investigate the dynamics of the masses of each component, i.e., $\mathcal{M}_{u}^{n}-\mathcal{M}_{u}^{0}, \mathcal{M}_{v}^{n}-\mathcal{M}_{v}^{0}$ and the total mass $\mathcal{M}^{n}-\mathcal{M}^{0}$ with $\mathcal{M}^{n}=\mathcal{M}_{u}^{n}+\mathcal{M}_{v}^{n}$. The contours of the densities $\left|u_{j k}^{n}\right|^{2}$ and $\left|v_{j k}^{n}\right|^{2}$ at different moments $t=0,6,12,18,24,30$ are depicted in Fig. 1 and Fig. 2, respectively. Fig. 3 graphs the error evolution of energy, component mass and total mass with time.

From these pictures, we can observe that the density functions $\left|u_{j k}^{n}\right|^{2}$ and $\left|v_{j k}^{n}\right|^{2}$ are Gaussian distribution from the beginning to the end except that their amplitudes decrease and areas expand. The fluctuation range of energy is within machine precision which indicates discrete energy-preserving. This is consistent with our theoretical analysis. Although the mass of each component is not conserved, the oscillating amplitude is within a relatively small range. Moreover, the total mass is basically conserved.

Example 4.2 (Dynamics driven by rotation $(\Omega \neq 0)$ and free of internal atomic Josephson junction $(\lambda=0)$ ). In this example, we consider the dynamic behavior of the macroscopic wave functions $u(x, y, t)$ and $v(x, y, t)$ driven by rotation and free of internal atomic Josephson junction, i.e., $\Omega=0.6, \lambda=0$. We take the interaction parameters between the two components $\beta_{11}=9.7, \beta_{22}=10.3, \beta_{12}=\beta_{21}=70$. We consider the problem in the spatial domain $[-12,12]^{2}$. We use the HOC-AVF scheme (2.11a)-(2.11b) to compute the problem till $t=60$ with mesh size $h_{x}=h_{y}=0.2$ and time step length $\tau=0.001$. In Figs. 4-5, 

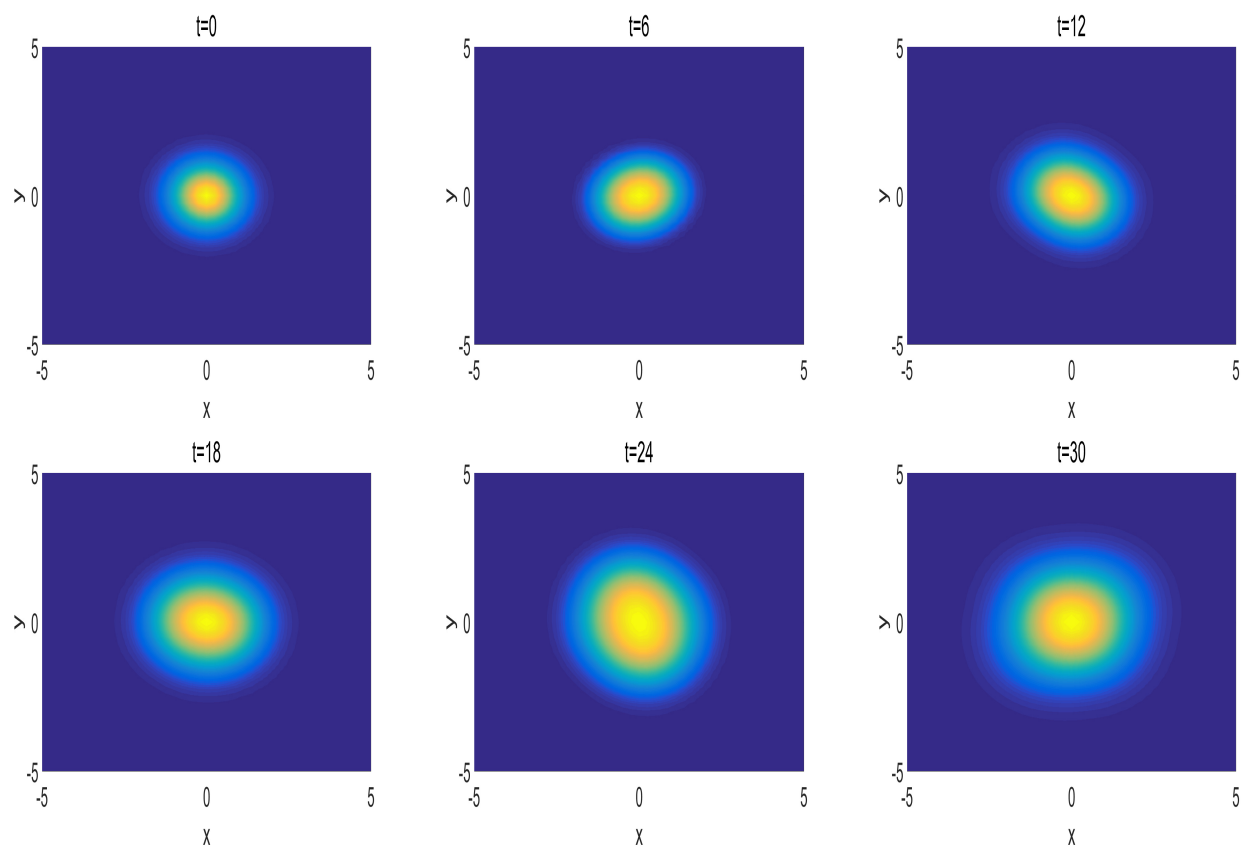

Figure 1: Contour plots of $\left|u_{j k}^{n}\right|^{2}$ at different times in Example 4.1.
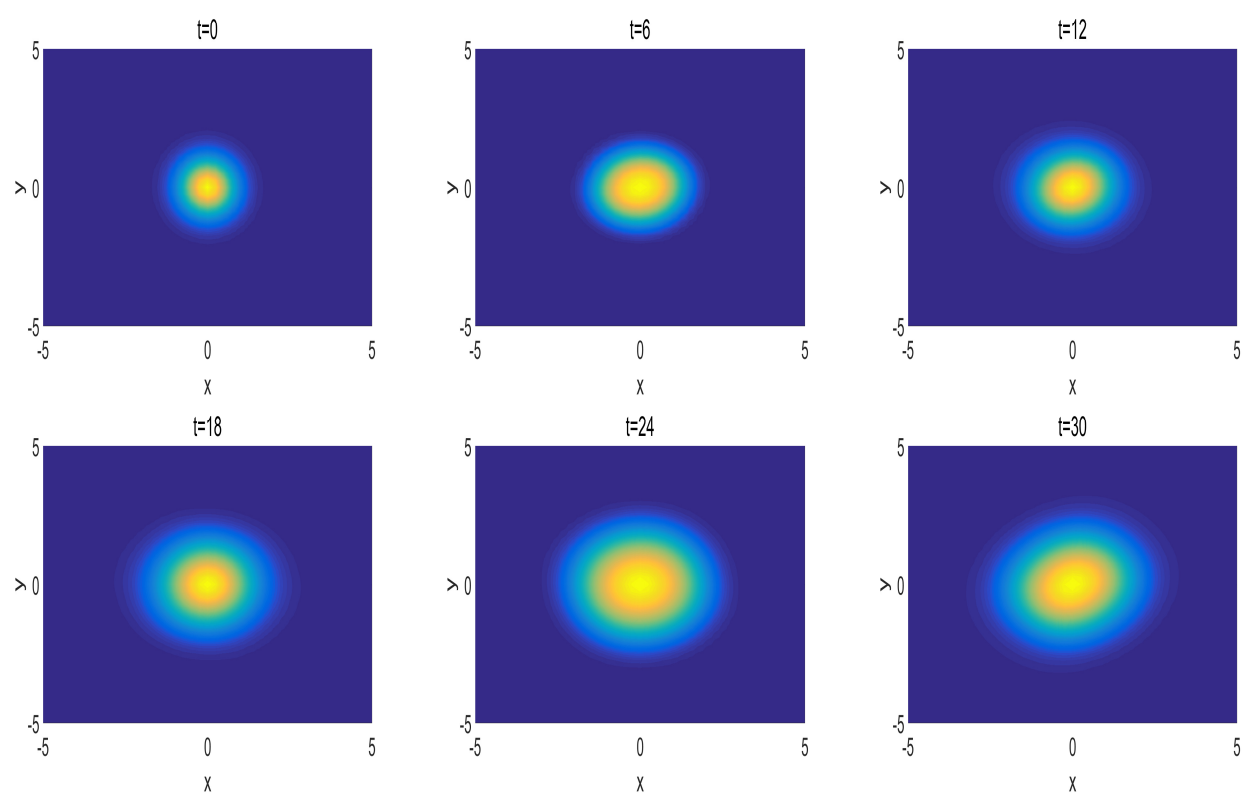

Figure 2: Contour plots of $\left|v_{j k}^{n}\right|^{2}$ at different times in Example 4.1. 

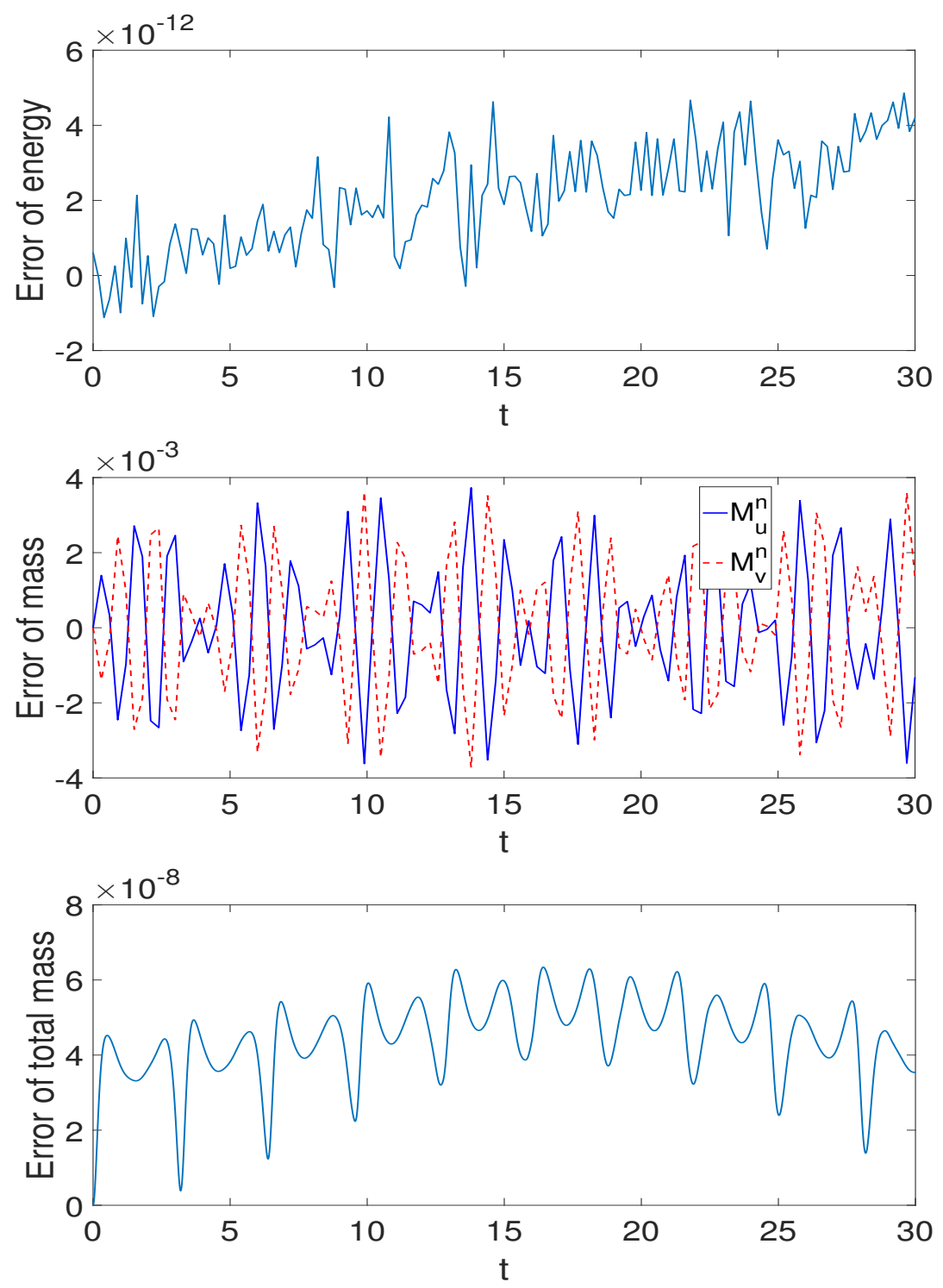

Figure 3: The evolution of energy $\mathcal{H}^{n}$ (top), component mass $\mathcal{M}_{u}{ }^{n}, \mathcal{M}_{v}{ }^{n}$ (middle) and total mass $\mathcal{M}^{n}$ (bottom) in Example 4.1.

we present the contour plots of $\left|u_{j k}^{n}\right|^{2}$ and $\left|v_{j k}^{n}\right|^{2}$ at $t=0,12,24,36,48,60$ to observe the evolution of the densities. The evolution of the residuals of energy, component-mass and total mass are plotted in Fig. 6.

From these figures, one can observe that the $u$-wave splits into two smaller Gaussian pluses immediately, while the $v$-wave keeps single wave along. As is expected, the energy is exactly preserved, the fluctuations of component mass and total mass jump in a 


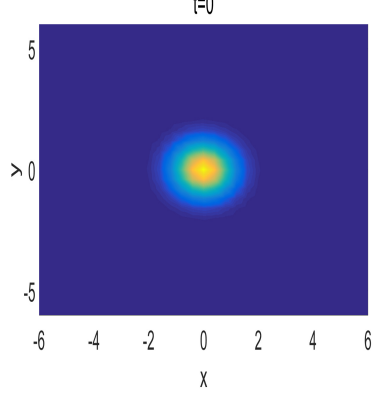

$t=36$

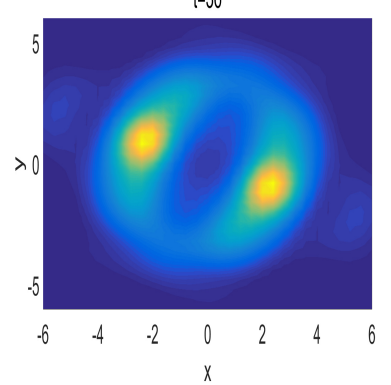

$t=12$

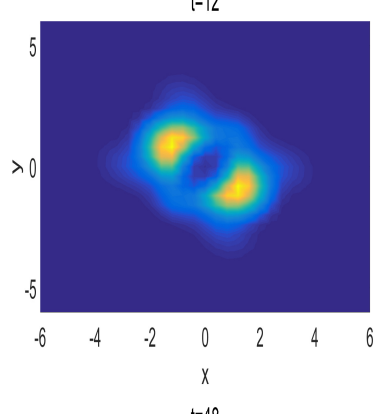

$t=48$

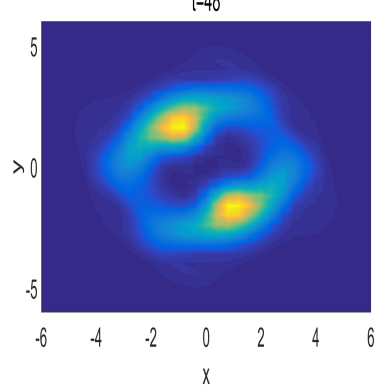

$t=24$

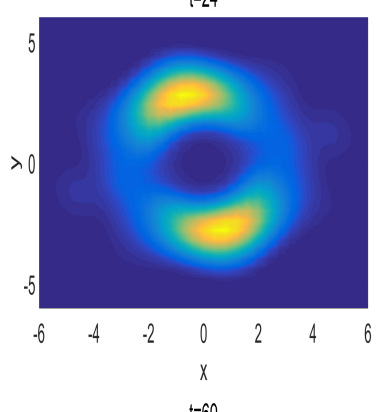

$t=60$

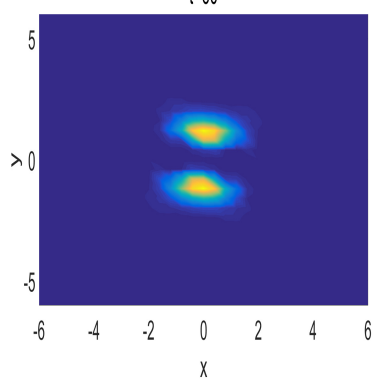

Figure 4: Contour plots of $\left|u_{j k}^{n}\right|^{2}$ at different times in Example 4.2.
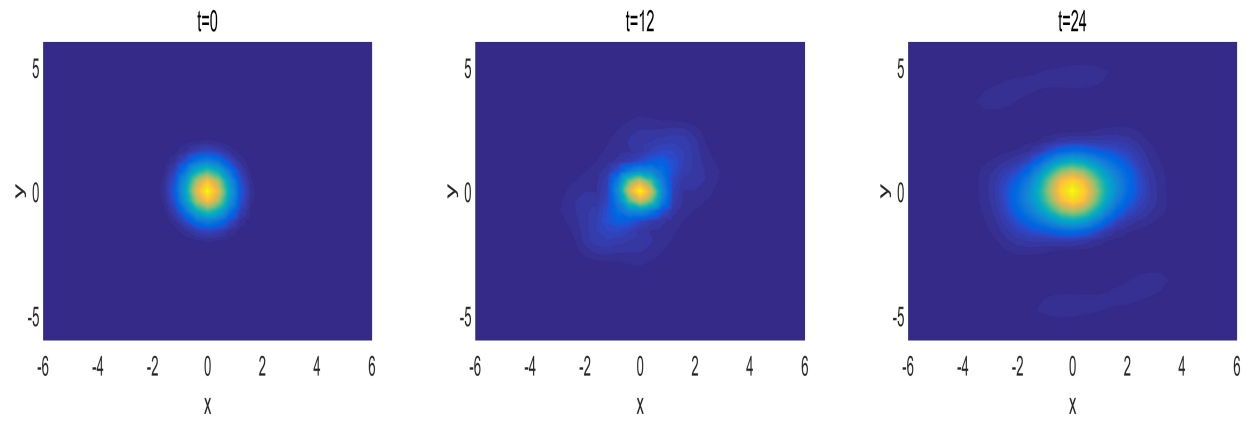

$t=36$
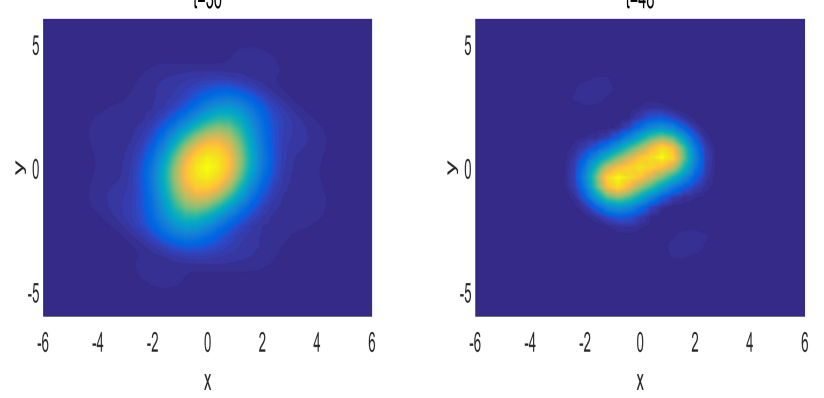

$t=60$

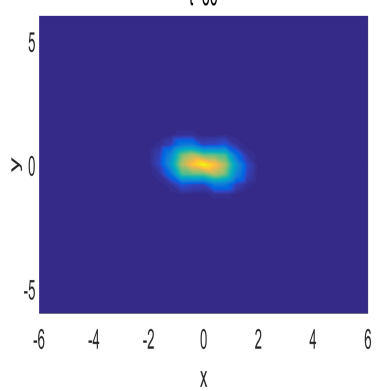

Figure 5: Contour plots of $\left|v_{j k}^{n}\right|^{2}$ at different times in Example 4.2. 

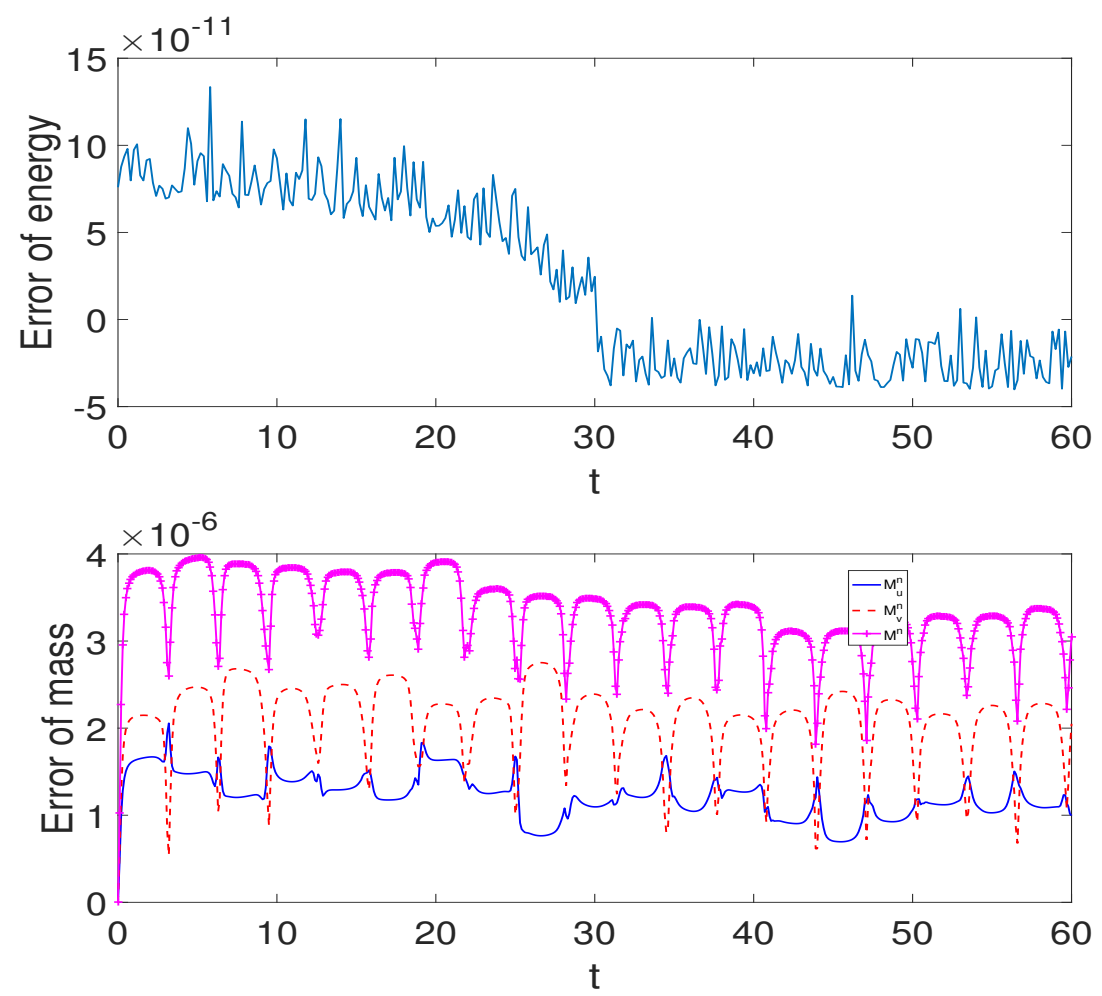

Figure 6: The error evolution of energy $\mathcal{H}^{n}$ (top) and mass $\mathcal{M}_{u}{ }^{n}, \mathcal{M}_{v}{ }^{n}, \mathcal{M}^{n}$ (bottom) in Example 4.2.

narrow interval.

Example 4.3 (Without rotation). In this example, we consider the dynamic behavior of the macroscopic wave functions $u(x, y, t)$ and $v(x, y, t)$ free of rotation, that is, $\Omega=0$. We choose the parameters $\lambda=0.5, \beta_{11}=9.7, \beta_{22}=10.3, \beta_{12}=\beta_{21}=10$. The problem is considered in the spatial square $[-7,7]^{2}$. We use the HOC-AVF scheme (2.11a)-(2.11b) to compute the problem with mesh size $h_{x}=h_{y}=0.1$ and $\tau=0.001$. The simulation time length is $T=20$.

In Figs. 7-8, we present the contour plots of $\left|u_{j k}^{n}\right|^{2}$ and $\left|v_{j k}^{n}\right|^{2}$ at different times to observe the evolution of the densities. Fig. 9 plots the evolution of the errors of energy, component mass and total mass over time.

From these figures, we have the following numerical observations:

- The evolutions of the densities of both components are always keeping Gaussian except the amplitude.

- The energy is preserved exactly from the beginning to the end, which is consistent with our expectation. 


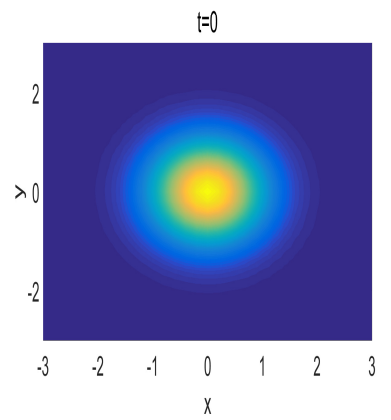

$t=12$

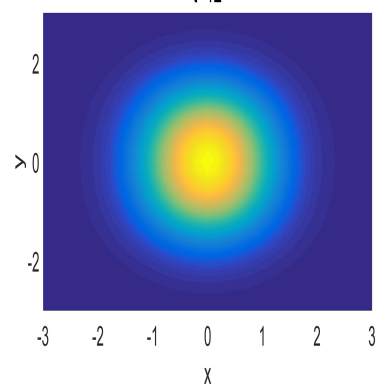

Figure 7: Contour plots of $\left|u_{j k}^{n}\right|^{2}$ at different times in Example 4.3.

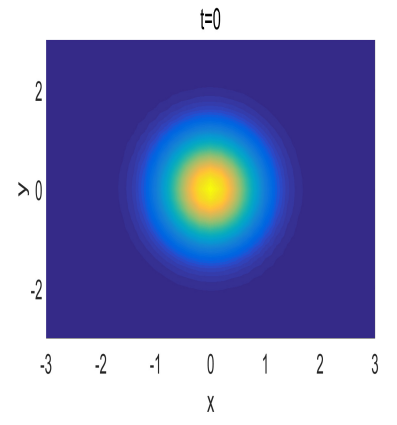

$t=12$

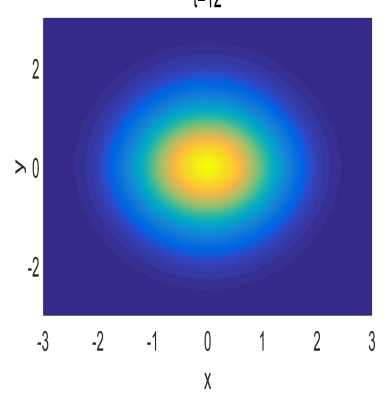

$t=4$

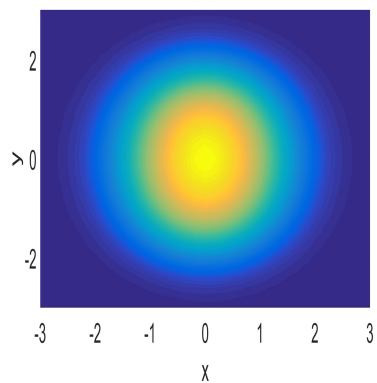

$\mathrm{t}=16$

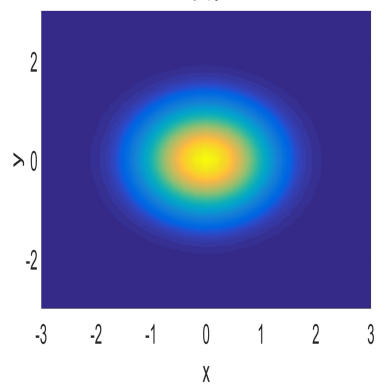

$t=4$

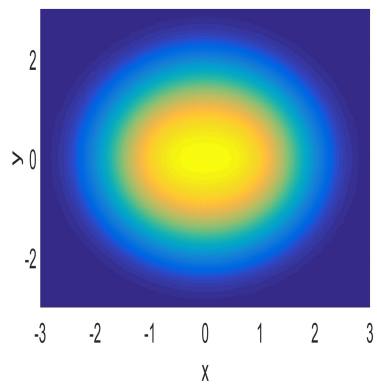

$t=16$

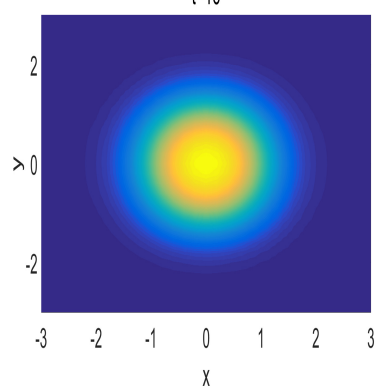

$t=8$

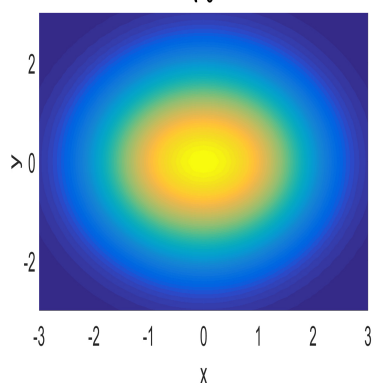

$t=20$

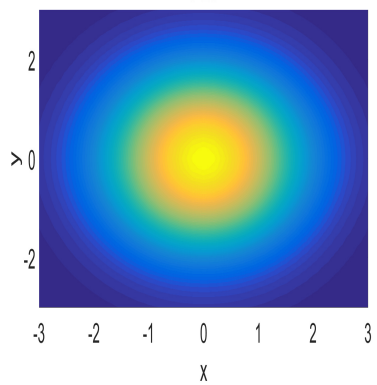

$t=8$

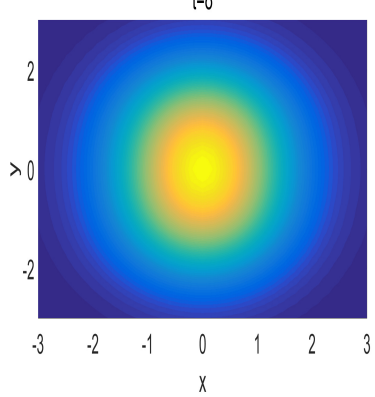

$t=20$

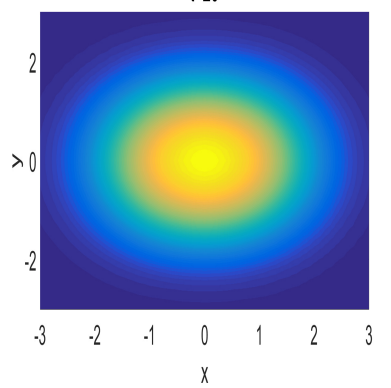

Figure 8: Contour plots of $\left|v_{j k}^{n}\right|^{2}$ at different times in Example 4.3. 

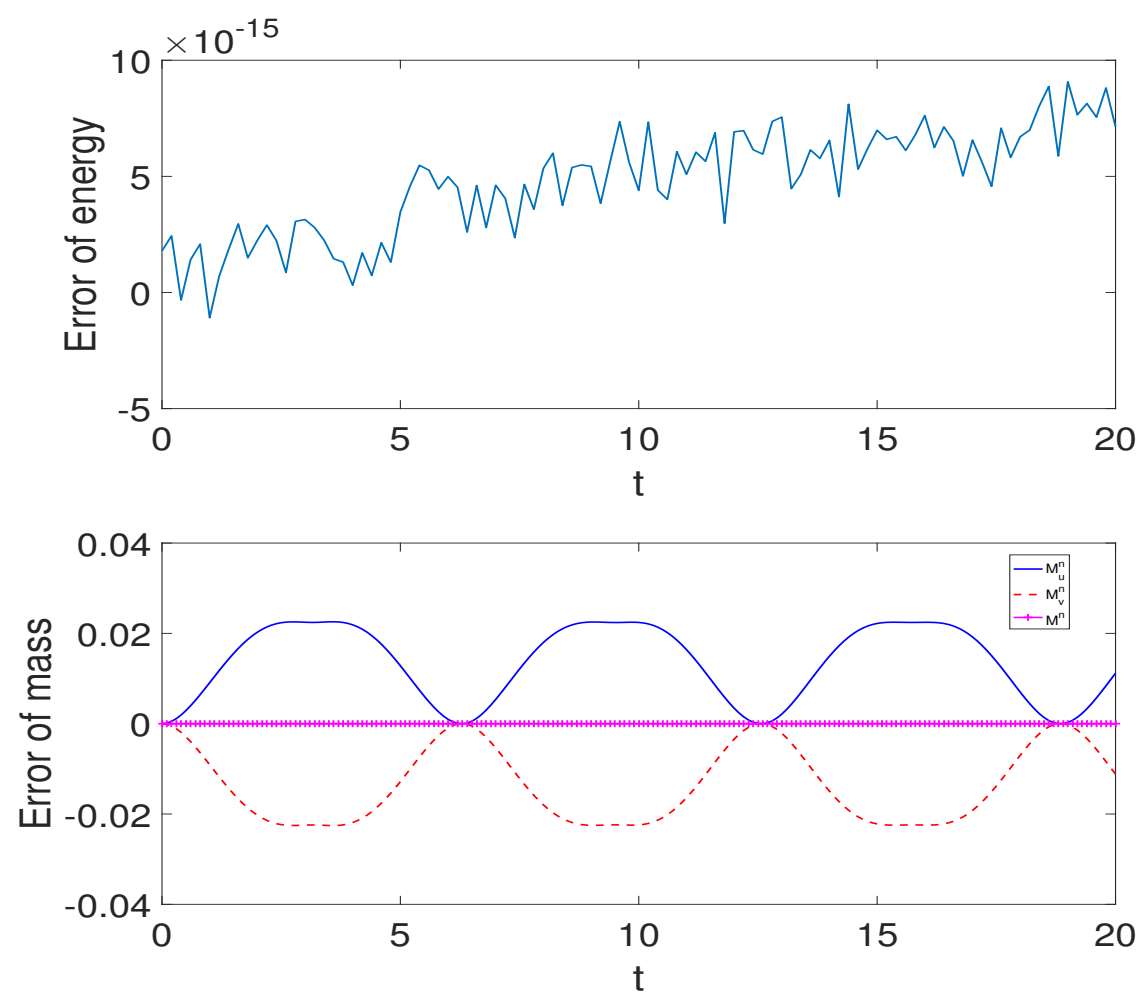

Figure 9: Time evolution of energy $\mathcal{H}^{n}$ (top) and mass $\mathcal{M}_{u}{ }^{n}, \mathcal{M}_{v}{ }^{n}, \mathcal{M}^{n}$ (bottom) in Example 4.3.

- From the shape of the error of component mass $\mathcal{M}_{u}{ }^{n}$ and $\mathcal{M}_{v}{ }^{n}$, we can know that the mass-lossy in $u$-component is just compensated by the mass-obtain in $v$ component. Therefore, we can conjecture that the total mass is basically preserved.

Example 4.4 (Dynamics of quantized vortex lattices). In this example, we are interested in investigating the quantized vortex lattices in BECs described by the CGP equations (1.1a)-(1.1b) with the parameters $\beta_{11}=97, \beta_{22}=103, \beta_{12}=\beta_{21}=100, \lambda=0, \Omega=0.9$ and the quadratic potential function $s_{1}(x, y)=s_{2}(x, y)=\frac{1}{2}\left(x^{2}+y^{2}\right)$. The initial data used for this example are ground state of the rotating two-component GPEs at equilibrium, which are obtained by minimizing the energy functional of the system at equilibrium

$$
\begin{aligned}
\mathcal{E}(u, v)=\int_{D} & {\left[\frac{1}{2}\left(|\nabla u|^{2}+|\nabla v|^{2}\right)+s_{1}|u|^{2}+s_{2}|v|^{2}+\frac{1}{2} \beta_{11}|u|^{4}\right.} \\
+ & \left.\beta_{12}|u|^{2}|v|^{2}+\frac{1}{2} \beta_{22}|v|^{4}+\Omega\left(\bar{u} L_{z} u+\bar{v} L_{z} v\right)\right] d x d y .
\end{aligned}
$$

We set the spatial domain be the square $D=[-8,8]^{2}$. This ground state is computed by the backward Euler centered finite difference method and coded by the Matlab toolbox 


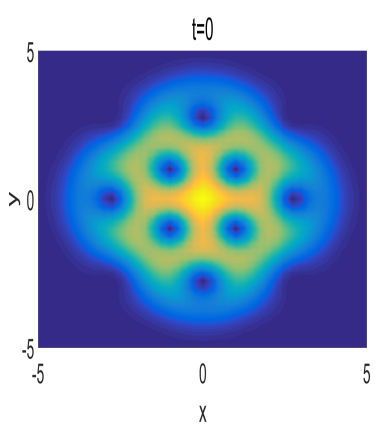

$t=6$
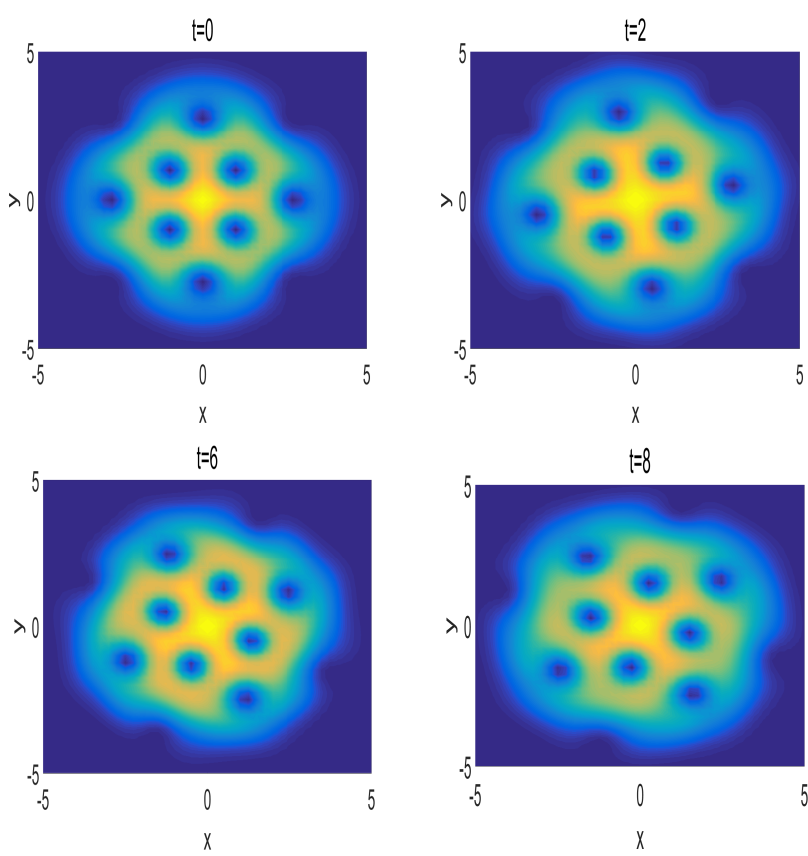

$t=8$

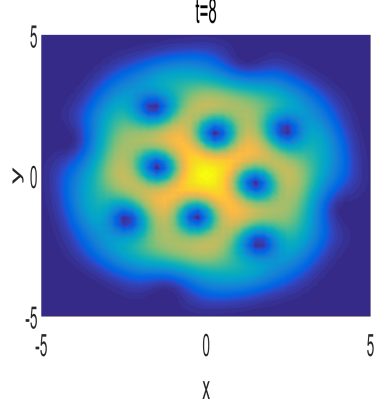

$t=4$

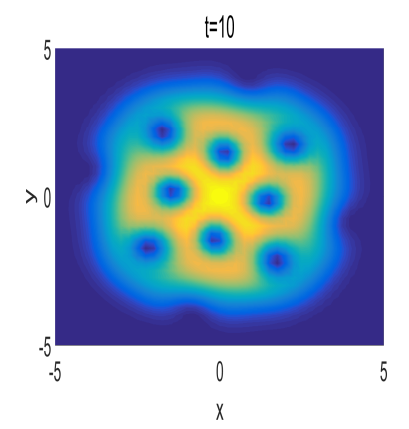

Figure 10: Contour plots of $\left|u_{j k}^{n}\right|^{2}$ at different times in Example 4.4.
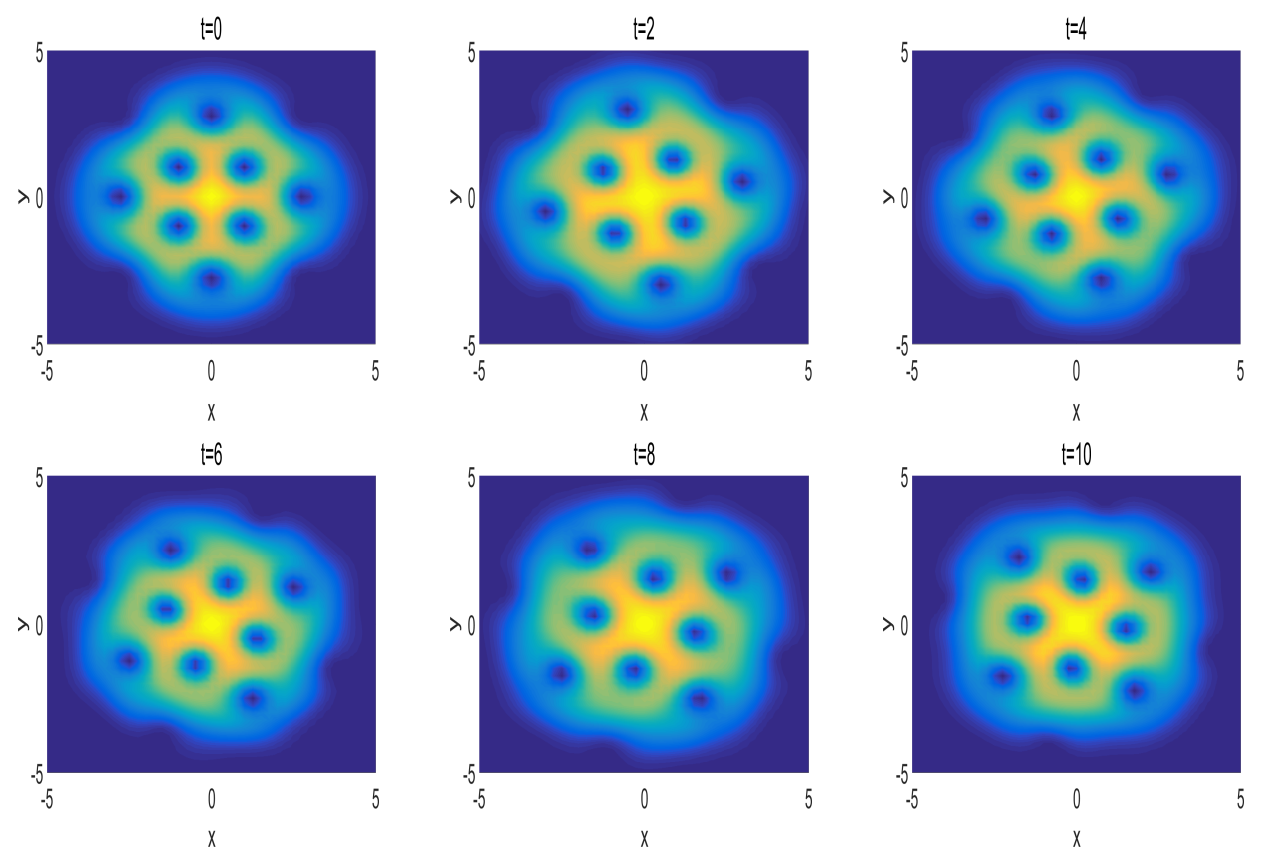

Figure 11: Contour plots of $\left|v_{j k}^{n}\right|^{2}$ at different times in Example 4.4. 

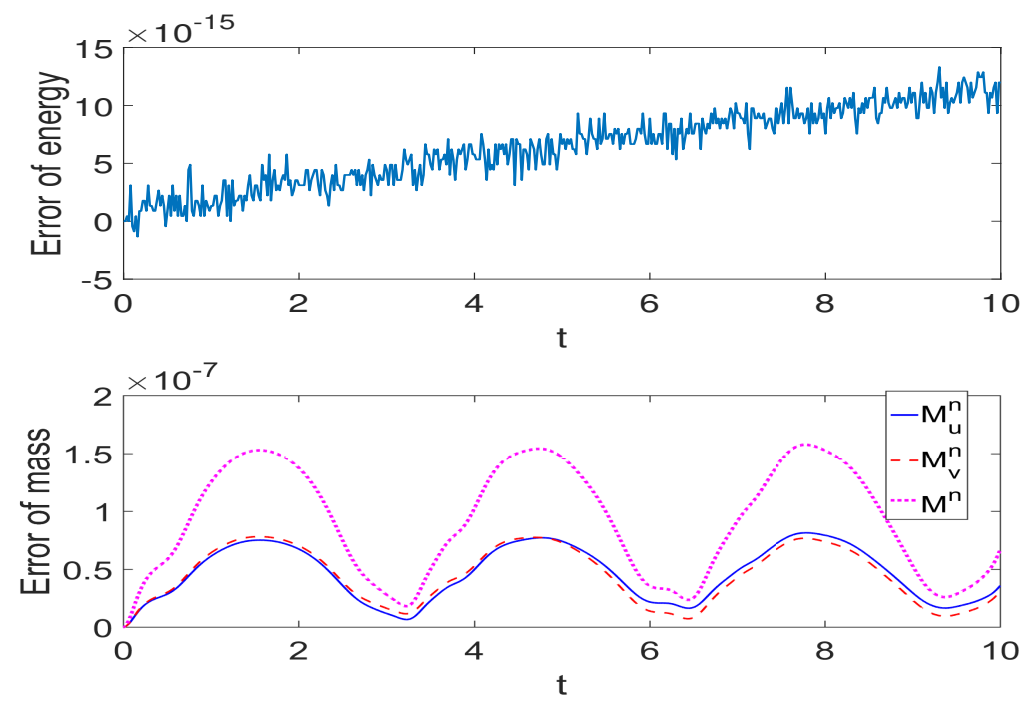

Figure 12: Time evolution of energy $\mathcal{H}^{n}$ (top) and mass $\mathcal{M}_{u}{ }^{n}, \mathcal{M}_{v}{ }^{n}, \mathcal{M}^{n}$ (bottom) in Example 4.4.

provided by Antoine [2]. We simulate the quantized vortex latices by the HOC-AVF scheme (2.11a)-(2.11b) till $t=10$ under partition $h_{x}=h_{y}=0.125, \tau=0.001$. Figs. 10-11 show the the contour plots of the density functions $u_{j k}^{n}$ and $v_{j k}^{n}$ at different times, while Fig. 12 presents the evolution of the residuals of energy and component mass and total mass.

From these figures, we can see that:

- All the vortex lattices rotate around the origin symmetrically with a similar pattern to the initial vortex lattices.

- The number (about 8) of the vortex lattices is preserved during the whole dynamic simulation.

- The energy is exactly preserved up to machine precision. The residual of the mass of each component is very small from the beginning to the end.

\section{Conclusions and remarks}

In this manuscript, an energy-preserving scheme is proposed for the CGP equations. High order compact method and average vector field method are applied in the spatial and the temporal directions, respectively. Then we obtain a sixth order in space and second order in time energy-preserving scheme. The error estimates of this scheme are obtained by energy method. Various numerical results in different cases support our theoretical analysis. 


\section{Acknowledgements}

This work is supported by the National Natural Science Foundation of China (Nos. 11771213, and 11961036), the Natural Science Foundation of Jiangxi Province (Nos. 20161ACB20006, 20142BCB23009, and 20181BAB201008). The authors are grateful to the anonymous referees for their invaluable suggestions in improving this paper.

\section{References}

[1] Y. ADAM, Highly accurate compact implicit methods and boundary conditions, J. Comput. Phys., 24 (1977), pp. 10-22.

[2] X. AnOINE, AND R. DuboscQ, GPELab, a Matlab toolbox to solve Gross-Pitaevskii equations I: Computation of stationary solution, Comput. Phys. Commun., 185 (2014), pp. 2969-2991.

[3] W. Z. BAO AND Y. Y. CAI, Mathematical theory and numerical methods for Bose-Einstein condensation, Kinet. Relat. Mod., 6 (2013), pp. 1-135.

[4] W. Z. BAO AND Y. Y. CAI, Optimal error estimates of finite difference methods for the GrossPitaevskii equations with angular momentum rotation, Math. Comput., 82 (2013), pp. 99-128.

[5] W. Z. BAO, Q. DU AND Y. Z. ZHANG, Dynamics of rotating Bose-Einstein condensates and their efficient and accurate numerical computation, SIAM J. Appl. Math., 66 (2006), pp. 758-786.

[6] W. Z. BAO, H. L. LI AND J. SHEN, A generalized Laguerre-Fourier-Hermite pseudospectral method for computing the dynamics of rotating Bose-Einstein condensates, SIAM J. Sci. Comput., 31 (2009), pp. 3685-3711.

[7] J. X. CAI, Y. S. WANG AND Y. Z. GONG, Numerical analysis of AVF schemes for threedimensional time-domain Maxwell's equations, J. Sci. Comput., 66 (2016), pp. 141-176.

[8] W. J. CAI, H. C. LI AND Y. S. WANG, Partitioned averaged vectored field methods, J. Comput. Phys., 370 (2018), pp. 25-42.

[9] M. M. Cerimele, M. L. Chiofalo, F. Pistella, S. Succi AND M. Tosi, Numerical solution of the Gross-Pitaevskii equation using an explicit finite difference scheme: An application to trapped Bose-Einstein condensates, Phys. Rev. E, 62 (2000), pp. 1382-1389.

[10] M. CHEN, L. H. KONG AND Y. Q. HONG, Efficient structure-preserving schemes for good Boussinesq equation, Math. Meth. Appl. Sci., 41 (2018), pp. 1743-1752.

[11] K. B. Davis, M. O. Mewes, M. R. Andrews, N. J. Druten, D. S. Durfee, D. M. Kurn AND W. KeTterle, Bose-Einstein condensation in a gas of sodium atoms, Phys. Rev. Lett., 75 (1995), pp. 3969-3973.

[12] F. F. FU, L. H. KONG, L. WANG AND Y. XU, High order compact splitting multisymplectic schemes for 1D Gross-Pitaerskii equation, Chin. J. Comput. Phys., 35 (2018), pp. 607-617.

[13] Q. HONG, Y. S. WANG AND Q. K. DU, Two new energy-preserving algorithms for generalized fifth-order KdV equation, Adv. Appl. Math. Mech., 9 (2017), pp. 1206-1224.

[14] L. H. KONG, Y. L. DUAN, L WANG AND Y. P. MA, Spectral-like resolution compact ADI finite difference method for the multi-dimensional Schrödinger equations, Math. Comput. Model., 55 (2012), pp. 1798-1812.

[15] L. H. Kong, J. L. Hong AND J. J. ZHANG, LOD-MS for Gross-Pitaerskii equation in BoseEinstein condensates, Commun. Comput. Phys., 14 (2013), pp. 219-241.

[16] K. Kasamatsu, M. Tsubota AND M. UedA, Vortices in multicomponent Bose-Einstein condensates, Int. J. Modern Phys. B, 19 (2005), pp. 1835-1904. 
[17] R. LANDES, On Galerkin's method in the existence theory of quasilinear elliptic equations, J. Funct. Anal., 39 (1980), pp. 123-148.

[18] M. C. LAI AND C. Y. HuANG, A simple Dufort-Frankel type scheme for the Gross-Pitaerskii equation of Bose-Einstein condensates on different geometries, Numer. Meth. Partial Differential Equations, 20 (2004), pp. 624-638.

[19] J. C. LI, High order compact ADI methods for the parabolic equations, Comput. Math. Appl., 52 (2006), pp. 1343-1356.

[20] S. K. LELE, Compact finite difference schemes with spectral-like solution, J. Comput. Phys., 103 (1992), pp. 16-42.

[21] Y. P. MA, L. H. KONG AND J. L. HONG, High-order compact splitting multisymplectic method for the coupled nonlinear Schrödinger equation, Comput. Math. Appl., 61 (2011), pp. 319-333.

[22] K. W. Madison, F. Chevy, W. Wohlleben AND J. Dalibard, Vortex formation in a stirred Bose-Einstein condensate, Phys. Rev. Lett., 84 (2000), pp. 806-809.

[23] M. R. Matthews, B. P. Anderson, P. C. Haljan, D. S. Hall, C. E. Wieman and E. A. CORNEII, Vortices in a Bose-Einstein condensate, Phys. Rev. Lett., 83 (1999), pp. 201-202.

[24] R. J. Mclachlan, G. R. W. QueisPel and N. Robidoux, Geometric integration using discrete gradients, Phil. Trans. R. Soc., 357 (1999), pp. 1021-1046.

[25] M. Modugno, F. Dalfovo, C. Fort, P. Maddaloni and F. Minardi, Dynamics of two colliding Bose-Einstein condensates in an elongated magnetosatic trap, Phys. Rev. A, 62 (2000), pp. 063607.

[26] J. Ming, Q. L. TANG AND Y. Z. ZHANG, An efficient spectral method for computing dynamics of rotating two-component Bose-Einstein condensates via coordinate transformation, J. Comput. Phys., 258 (2014), pp. 538-554.

[27] Z. G. MU, H. C. LI, Y. S. WANG AND W. J. CAI, A Galerkin splitting symplectic method for the two dimensional nonlinear Schrödinger equation, Adv. Appl. Math. Mech., 10 (2018), pp. 1069-1089.

[28] G. R. W. QuisPEL AND D. I. MCLAREN, A new class of energy-preserving numerical integration methods, J. Phys. A Math. Theor., 41 (2008), 045206.

[29] Y. SHI, Q. MA AND X. H. DING, A new energy-preserving scheme for the fractional Klein-GordonSchrödinger equations, Adv. Appl. Math. Mech., 11 (2019), pp. 1219-1247.

[30] H. TONG, L. H. KONG AND L. WANG, Compact splitting multisymplectic scheme for Dirac equation, J. Jiangxi Normal Univer., 38 (2014), pp. 521-525.

[31] H. Q. WANG, A time-splitting spectral method for coupled Gross-Pitaevskii equations with applications to rotating Bose-Einstein condensates, J. Comput. Appl. Math., 205 (2007), pp. 88-104.

[32] T. C. WANG, B. L. GUO AND Q. XU, Fourth-order compact and energy conservative difference schemes for the nonlinear Schrödinger equation in two dimensions, J. Comput. Phys., 243 (2013), pp. 382-399.

[33] T. C. WANG, Optimal point-wise error estimate of a compact difference scheme for the coupled Gross-Pitaevskii equations in one dimension, J. Sci. Comput., 59 (2014), pp. 158-186.

[34] T. C. WANG AND X. F. ZHAO, Optimal $l^{\infty}$ error estimates of finite difference methods for the coupled Gross-Pitaevskii equations in high dimensions, Sci. China Math., 57 (2014), pp. 21892214.

[35] Y. Z. ZHANG, W. Z. BAO AND H. L. LI, Dynamics of rotating two-component Bose-Einstein condensates and its efficient computation, Phys. D Nonlin. Phenon., 234 (2007), pp. 49-69.

[36] Y. L. ZHOU, Application of Discrete Functional Analysis to the Finite Difference Method, Beijing: International Academic Publishers, 1990. 